LHCb 2000-002, PHYS

(IPHE 2000-006)

February 112000

\title{
PHYSICS OF CP VIOLATION AND RARE DECAYS
}

\author{
Lectures note for Cargese 99 Summer School \\ Particle Physics: Ideas and Recent Development \\ NATO Advanced Institute, Corsica, July 26-August 7, 1999
}

\author{
T. NAKADA \\ CERN EP-Division \\ CH-1211 Geneva 23, Switzerland \\ and
}

Institute of High Energy Physics, University of Lausanne

CH-1015 Dorigny, Switzerland

(On leave from PSI, CH-5232 Villigen-PSI, Switzerland) 



\section{Introduction}

Symmetries are one of the most fundamental concepts for understanding the laws of nature leading to conserving quantities. Unexpected violations of symmetries indicate some dynamical mechanism beyond the current understanding of physics.

Parity violation was discovered in 1957 [四] in nuclear $\beta$ decays and pion and muon decays [2]. In the charged current interaction of the standard electroweak theory, parity and charge conjugation symmetries are maximally violated due to the $V-A$ structure [3]. All the experimental results up to now are in full agreement with the theory.

A surprising discovery of the $\mathrm{CP}$ violating $\mathrm{K}_{\mathrm{L}} \rightarrow \pi^{+} \pi^{-}$decays [4 was made in 1964. The neutral kaon system still remains to be the only place $\mathrm{CP}$ violation has been seen. The Standard Model with three Fermion families can accommodate all the observed $\mathrm{CP}$ violation phenomena through the complex quark mixing matrix, Cabibbo-Kobayashi-Maskawa (CKM) matrix [5]. However, no real precision test has been made due to the large uncertainties in evaluating the effect of hadronic interactions.

Interest in $\mathrm{CP}$ violation is not limited to elementary particle physics. It is one of the three necessary ingredients to generate observed excess of matter over antimatter in the universe [6]. The amount of CP violation which can be generated by the Standard Model appears to be insufficient for explaining the observed matter-antimatter asymmetry in the universe [7], giving a strong motivation to search for new physics.

For CP violation in some B meson decay channels, the Standard Model can make precise predictions with little influence from the strong interactions. Those channels can be used to test the predictions quantitatively to look for a sign of new physics. Also in the $\mathrm{B}$ meson system, CP violation is expected in many decay modes. The pattern of CP violation allows us to make a systematic qualitative comparison with the Standard Model predictions. Therefore, it is now widely accepted that the Bmeson system provides in future an ideal place for testing the Standard Model for CP violation [9].

In this article, we first derive the formalism [10] describing the particle antiparticle system, with and without $\mathrm{CP}$ violation. Three different mechanisms which can

generate CP violation are clearly classified, together with experimental observables which identify contributions from the different mechanisms. Then, $\mathrm{CP}$ violation in the neutral kaon system is analysed in this formalism. After a brief discussion on the Standard Model description for CP violation in the neutral kaon system, we proceed to the neutral B meson system. Following the discussion on some Standard Model predictions, some thoughts are made how the situation could change if there exists new physics contributing in the B meson system. 


\section{Description of Particle Antiparticle System}

\subsection{Basic Formalism}

Let $\left|\mathrm{P}^{0}\right\rangle$ and $\left|\overline{\mathrm{P}}^{0}\right\rangle$ be the states of a neutral pseudoscalar particle $\mathrm{P}^{0}$-meson and its antiparticle $\overline{\mathrm{P}}^{0}$-meson at rest, respectively. They have definite flavour quantum numbers with opposite signs: $F=+1$ for $\mathrm{P}^{0}$ and $F=-1$ for $\overline{\mathrm{P}}^{0}$. Both states are eigenstates of the strong and electromagnetic interaction Hamiltonian, i.e.

$$
\left(H_{\mathrm{st}}+H_{\mathrm{em}}\right)\left|\mathrm{P}^{0}\right\rangle=m_{0}\left|\mathrm{P}^{0}\right\rangle \text { and }\left(H_{\mathrm{st}}+H_{\mathrm{em}}\right)\left|\overline{\mathrm{P}}^{0}\right\rangle=\bar{m}_{0}\left|\overline{\mathrm{P}}^{0}\right\rangle
$$

where $m_{0}$ and $\bar{m}_{0}$ are the rest masses of $\mathrm{P}^{0}$ and $\overline{\mathrm{P}}^{0}$, respectively. The $\mathrm{P}^{0}$ and $\overline{\mathrm{P}}^{0}$ states are related through $\mathrm{CP}$ transformations. For stationary states, the $\mathrm{T}$ transformation does not alter them, with the exception of an arbitrary phase. While CP is a unitary operation, $\mathrm{T}$ is an antiunitary operation.

In summary, we obtain

$$
\begin{gathered}
C P\left|\mathrm{P}^{0}\right\rangle=e^{i \theta_{\mathrm{CP}}}\left|\overline{\mathrm{P}}^{0}\right\rangle \text { and } C P\left|\overline{\mathrm{P}}^{0}\right\rangle=e^{-i \theta_{\mathrm{CP}}}\left|\mathrm{P}^{0}\right\rangle \\
T\left|\mathrm{P}^{0}\right\rangle=e^{i \theta_{\mathrm{T}}}\left|\mathrm{P}^{0}\right\rangle \text { and } T\left|\overline{\mathrm{P}}^{0}\right\rangle=e^{i \bar{\theta}_{\mathrm{T}}}\left|\overline{\mathrm{P}}^{0}\right\rangle
\end{gathered}
$$

where the $\theta$ 's are arbitrary phases, and by assuming $C P T\left|\mathrm{P}^{0}\right\rangle=T C P\left|\mathrm{P}^{0}\right\rangle$ it follows that

$$
2 \theta_{\mathrm{CP}}=\bar{\theta}_{\mathrm{T}}-\theta_{\mathrm{T}}
$$

Since $\mathrm{T}$ is antiunitary, it follows that

$$
T c=c^{*} T
$$

where $c$ is any complex number. If we define

$$
T|\alpha\rangle=|\tilde{\alpha}\rangle, T|\beta\rangle=|\tilde{\beta}\rangle
$$

antiunitary operation has to give

$$
\langle\alpha \mid \beta\rangle=[\langle\tilde{\alpha} \mid \tilde{\beta}\rangle]^{*}
$$

On the other hand,

$$
\langle\alpha \mid \beta\rangle=\langle\alpha|\left(T^{-1} T|\beta\rangle\right)=\langle\alpha|\left(T^{-1}|\tilde{\beta}\rangle\right),
$$

hence

$$
\langle\alpha|\left(T^{-1}|\tilde{\beta}\rangle\right)=[\langle\tilde{\alpha} \mid \tilde{\beta}\rangle]^{*}
$$


We can then conclude

$$
\langle\alpha|\left(T^{-1}|\tilde{\beta}\rangle\right)=\left[\left(\langle\alpha| T^{-1}\right)|\tilde{\beta}\rangle\right]^{*}
$$

i.e. when the $\mathrm{T}$ operator changes the direction of the operation, it must be complex conjugated.

If strong and electromagnetic interactions are invariant under the CPT transformation, which is assumed throughout this paper, it follows that $m_{0}=\bar{m}_{0}$.

Now we switch on an interaction, $V$, and the $\mathrm{P}$ can decay into final states $\mathrm{f}$ with different flavours $(|\Delta F|=1$ process $)$ and $\mathrm{P}^{0}$ and $\overline{\mathrm{P}}^{0}$ can oscillate to each other $(|\Delta F|=2$ process). Thus, a general state $|\psi(t)\rangle$ which is a solution of the Schrödinger equation

$$
i \frac{\partial}{\partial t}|\psi(t)\rangle=\left(H_{\mathrm{st}}+H_{\mathrm{em}}+V\right)|\psi(t)\rangle
$$

can be written as

$$
|\psi(t)\rangle=a(t)\left|\mathrm{P}^{0}\right\rangle+b(t)\left|\overline{\mathrm{P}}^{0}\right\rangle+\sum_{\mathrm{f}} c_{\mathrm{f}}(t)|\mathrm{f}\rangle
$$

where the sum is taken over all the possible final states $\mathrm{f}$ and $a(t), b(t)$ and $c_{\mathrm{f}}(t)$ are time dependent functions; $|a(t)|^{2},|b(t)|^{2}$ and $\left|c_{\mathrm{f}}(t)\right|^{2}$ give the fractions of $\mathrm{P}^{0}, \overline{\mathrm{P}}^{0}$ and $\mathrm{f}$ at time $t$ respectively. Since the weak interaction is much weaker than strong and electromagnetic interactions, perturbation theory can be applied in order to solve equation 2. Also with the help of the Wigner-Weisskopf method, which neglects the weak interactions between the final states [8], and we obtain

$$
i \frac{\partial}{\partial t}\left(\begin{array}{c}
a(t) \\
b(t)
\end{array}\right)=\boldsymbol{\Lambda}\left(\begin{array}{c}
a(t) \\
b(t)
\end{array}\right)=\left(\boldsymbol{M}-i \frac{\boldsymbol{\Gamma}}{2}\right)\left(\begin{array}{c}
a(t) \\
b(t)
\end{array}\right)
$$

where the $2 \times 2$ matrices $\boldsymbol{M}$ and $\boldsymbol{\Gamma}$ are often referred to as the mass and decay matrices.

The elements of the mass matrix are given as

$$
M_{i j}=m_{0} \delta_{i j}+\langle i|V| j\rangle+\sum_{\mathrm{f}} \mathcal{P}\left(\frac{\langle i|V| \mathrm{f}\rangle\langle\mathrm{f}|V| j\rangle}{m_{0}-E_{\mathrm{f}}}\right)
$$

where $\mathcal{P}$ stands for the principal part and the index $i=1(2)$ denotes $\mathrm{P}^{0}\left(\overline{\mathrm{P}}^{0}\right)$. Note that the sum is taken over all possible intermediate states common to $\mathrm{P}^{0}$ and $\overline{\mathrm{P}}^{0}$ for $i \neq j$.

The elements of the decay matrix are given by

$$
\Gamma_{i j}=2 \pi \sum_{\mathrm{f}}\langle i|V| \mathrm{f}\rangle\langle\mathrm{f}|V| j\rangle \delta\left(m_{0}-E_{\mathrm{f}}\right)
$$

The sum is taken over only real final states common to $\mathrm{P}^{0}$ and $\overline{\mathrm{P}}^{0}$ for $i \neq j$. 
If the Hamiltonians are not Hermitian, transition probabilities are not conserved in decays or oscillations, i.e. the number of initial states is not identical to the number of final states. This is also referred to as the break down of unitarity. We assume from now on that all the Hamiltonians are Hermitian, i.e.

$$
|a(t)|^{2}+|b(t)|^{2}+\sum_{\mathrm{f}}\left|c_{\mathrm{f}}\right|^{2}=1
$$

and also

$$
M_{i j}=M_{j i}^{*}, \Gamma_{i j}=\Gamma_{j i}^{*} .
$$

Clearly $|a(t)|^{2}+|b(t)|^{2}$ decreases as a function of time, hence $\boldsymbol{\Lambda}$ is not Hermitian.

Since the CP operator changes a particle state into an antiparticle state, the following relation can be obtained if $V$ is invariant under the CP transformation, i.e. $(C P)^{-1} V C P=V$ :

$$
\mathrm{CP}: \quad\left|\Lambda_{12}\right|=\left|\Lambda_{21}\right|, \Lambda_{11}=\Lambda_{22} .
$$

Since the T operator induces complex conjugation, which is equivalent to interchanging a bra-state and a ket-state, the following relation can be obtained if $V$ is invariant under the $\mathrm{T}$ transformation:

$$
\mathrm{T}: \quad\left|\Lambda_{12}\right|=\left|\Lambda_{21}\right|
$$

By combining the two, we obtain for the CPT invariant case:

$$
\mathrm{CPT}: \Lambda_{11}=\Lambda_{22} \text {. }
$$

For a rigorous proof, equations 1, 4 and 5 are used.

It follows that

-if $\Lambda_{11} \neq \Lambda_{22}$, i.e. $M_{11} \neq M_{22}$ or $\Gamma_{11} \neq \Gamma_{22}$ :

CPT and CP are violated

-if $\left|\Lambda_{12}\right| \neq\left|\Lambda_{21}\right|$, i.e. $\sin \left(\varphi_{\Gamma}-\varphi_{M}\right) \neq 0$ :

$\mathbf{T}$ and $\mathbf{C P}$ are violated .

where $\varphi_{M}=\arg \left(M_{12}\right)$ and $\varphi_{\Gamma}=\arg \left(\Gamma_{12}\right)$. Note that CP violation in the mass and decay matrices cannot be separated from $\mathrm{CPT}$ violation or $\mathrm{T}$ violation.

While there is no fundamental reason to respect $\mathrm{CP}$ and $\mathrm{T}$ symmetries, it can be shown based on only few basic assumptions that no self consistent quantum field theory can be constructed that does not conserve CPT symmetry [11]. Therefore, we restrict our further discussion to the case where CPT symmetry is conserved: i.e.

$$
M_{11}=M_{22} \equiv M, \Gamma_{11}=\Gamma_{22} \equiv \Gamma
$$


thus

$$
\Lambda_{11}=\Lambda_{22} \equiv \Lambda
$$

Differential equation 3 can be reduced to

$$
\frac{d^{2} a(t)}{d t^{2}}+2 i \Lambda \frac{d a(t)}{d t}+\left(\Lambda_{12} \Lambda_{21}-\Lambda^{2}\right) a(t)=0
$$

for $a(t)$, and a general solution is given by

$$
a(t)=C_{+} e^{-i \lambda_{+}}+C_{-} e^{-i \lambda_{-}}
$$

where $C_{ \pm}$are arbitrary constants which can only be defined by the initial condition. For $b(t)$, we obtain

$$
b(t)=\frac{1}{\Lambda_{12}}\left[i \frac{d a(t)}{d t}-\Lambda a(t)\right]
$$

which can be used once $a(t)$ becomes known.

Insertion of $a(t)$ into equation 7 leads to

$$
\lambda_{ \pm}^{2}-2 \Lambda \lambda_{ \pm}+\left(\Lambda_{12} \Lambda_{21}-\Lambda^{2}\right)=0
$$

from which the eigen-frequencies are obtained as

$$
\lambda_{ \pm}=\Lambda \pm \sqrt{\Lambda_{12} \Lambda_{21}} \equiv m_{ \pm}-\frac{i}{2} \Gamma_{ \pm}
$$

by solving where

$$
m_{ \pm}=\Re \lambda_{ \pm}=M \pm \Re\left(\Lambda_{12} \Lambda_{21}\right)^{1 / 2}
$$

and

$$
\Gamma_{ \pm}=-2 \Im \lambda_{ \pm}=\Gamma \mp 2 \Im\left(\Lambda_{12} \Lambda_{21}\right)^{1 / 2} .
$$

For an initially pure $\mathrm{P}^{0}$ state, we have $a(t)=1$ and $b(t)=0$ at $t=0$, i.e. $C_{+}=C_{-}=1 / 2$, and the solution is given by

$$
\begin{aligned}
\left|\mathrm{P}^{0}(t)\right\rangle & =a(t)\left|\mathrm{P}^{0}\right\rangle+b(t)\left|\overline{\mathrm{P}}^{0}\right\rangle \\
& =f_{+}(t)\left|\mathrm{P}^{0}\right\rangle+\zeta f_{-}(t)\left|\overline{\mathrm{P}}^{0}\right\rangle \\
& =\frac{\sqrt{1+|\zeta|^{2}}}{2}\left(\left|\mathrm{P}_{+}\right\rangle e^{-i \lambda_{+} t}+\left|\mathrm{P}_{-}\right\rangle e^{-i \lambda_{-} t}\right)
\end{aligned}
$$

where

$$
f_{ \pm}(t)=\frac{1}{2}\left(e^{-i \lambda_{+} t} \pm e^{-i \lambda_{-} t}\right)
$$


and $\zeta$ is

$$
\zeta=\sqrt{\frac{\Lambda_{21}}{\Lambda_{12}}} .
$$

The two states $\left|\mathrm{P}_{+}\right\rangle$and $\left|\mathrm{P}_{-}\right\rangle$are the eigenstates of $\lambda_{ \pm}$and are given by

$$
\left|\mathrm{P}_{ \pm}\right\rangle=\frac{1}{\sqrt{1+|\zeta|^{2}}}\left(\left|\mathrm{P}^{0}\right\rangle \pm \zeta\left|\overline{\mathrm{P}}^{0}\right\rangle\right)
$$

For an initially pure $\overline{\mathrm{P}}^{0}$ state, we have

$$
\begin{aligned}
\left|\overline{\mathrm{P}}^{0}(t)\right\rangle & =\frac{1}{\zeta} f_{-}(t)\left|\mathrm{P}^{0}\right\rangle+f_{+}(t)\left|\overline{\mathrm{P}}^{0}\right\rangle \\
& =\frac{\sqrt{1+|\zeta|^{2}}}{2 \zeta}\left(\left|\mathrm{P}_{+}\right\rangle e^{-i \lambda_{+} t}-\left|\mathrm{P}_{-}\right\rangle e^{-i \lambda_{-} t}\right) .
\end{aligned}
$$

While $\mathrm{P}^{ \pm}$have definite masses and decay widths (as seen from equations 11 and [15), $\mathrm{P}^{0}$ and $\overline{\mathrm{P}}^{0}$ do not and they oscillate to each other (see equations 10 and [14).

\subsection{CP Conserving Case}

If $V$ remains invariant under the $\mathrm{CP}$ transformation, from equations 1 , 4 and 5 it follows that

$$
M_{12}=M_{21} e^{-i 2 \theta_{\mathrm{CP}}}=M_{12}^{*} e^{-i 2 \theta_{\mathrm{CP}}}
$$

thus

$$
\arg M_{12}=-\theta_{\mathrm{CP}}+n \pi,
$$

and

$$
\Gamma_{12}=\Gamma_{21} e^{-i 2 \theta_{\mathrm{CP}}}=\Gamma_{12}^{*} e^{-i 2 \theta_{\mathrm{CP}}}
$$

thus

$$
\arg \Gamma_{12}=-\theta_{\mathrm{CP}}+n^{\prime} \pi
$$

where $n$ and $n^{\prime}$ are arbitrary integer numbers.

For $\zeta$, we have

$$
\zeta=\sqrt{\frac{\Lambda_{21}}{\Lambda_{12}}}=e^{i\left(\theta_{\mathrm{CP}}+n^{\prime \prime} \pi\right)}
$$

where $n^{\prime \prime}$ is an arbitrary integer number. The two mass eigenstates $\left|\mathrm{P}_{+}\right\rangle$and $\left|\mathrm{P}_{-}\right\rangle$ become CP eigenstates

$$
C P\left|\mathrm{P}_{ \pm}\right\rangle= \pm(-1)^{n^{\prime \prime}}\left|\mathrm{P}_{ \pm}\right\rangle
$$

The mass and decay width eigenvalues, equations 8 and 9 , become

$$
m_{ \pm}=M \pm(-1)^{n+n^{\prime \prime}}\left|M_{12}\right|
$$




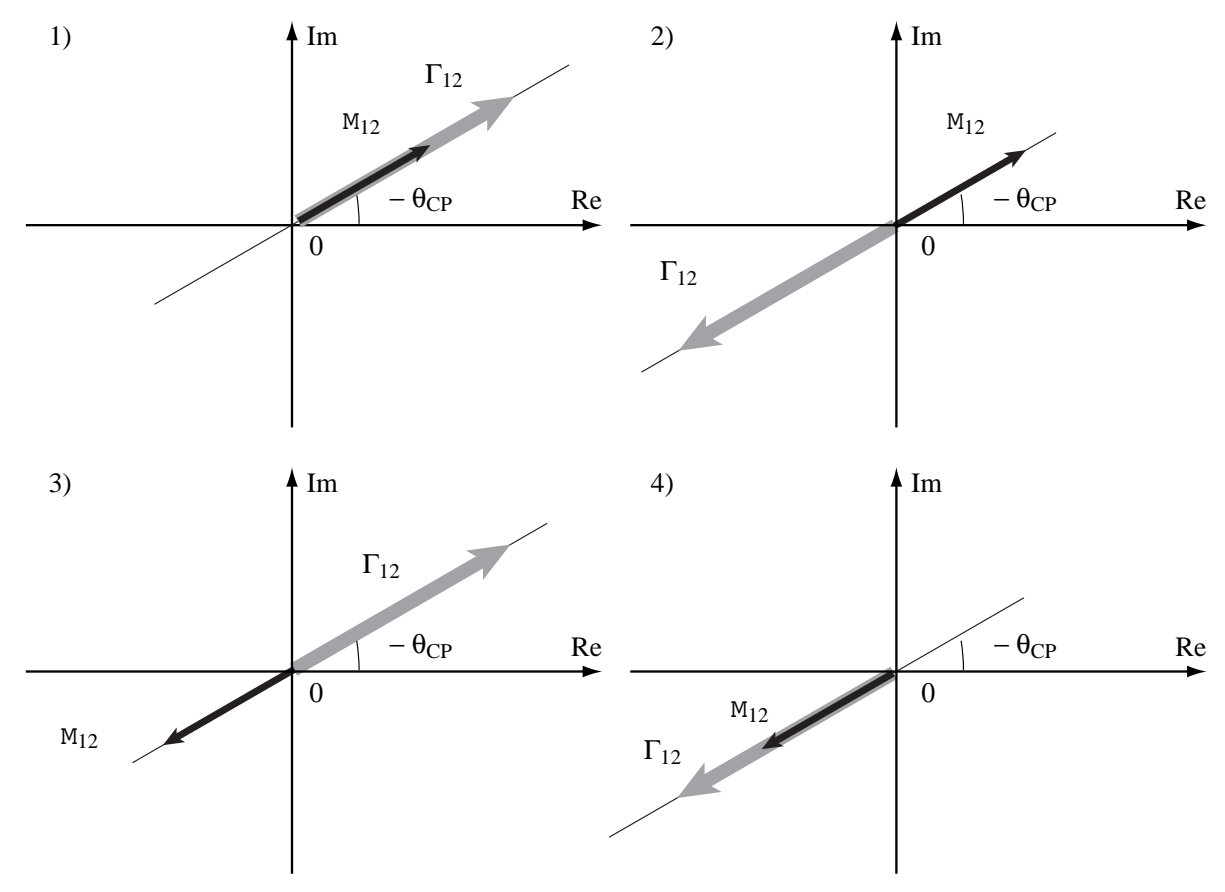

Figure 1: Relative phase relations for $M_{12}, \Gamma_{12}$, and CP transformation phase $\theta_{\mathrm{CP}}$ when $\mathrm{CP}$ is conserved: 1) $C P=+1$ state is heavier and decays faster, 2) $C P=+1$ state is heavier and decays slower, 3) $C P=+1$ state is lighter and decays faster, 4) $C P=+1$ state is lighter and decays slower.

and

$$
\Gamma_{ \pm}=\Gamma \pm(-1)^{n^{\prime}+n^{\prime \prime}}\left|\Gamma_{12}\right|
$$

By examining various combinations of $n, n^{\prime}$ and $n^{\prime \prime}$, we can show that the following four possibilities exist:

1. $n=$ even, $n^{\prime}=$ even: $C P=+1$ state is heavier and decays faster,

2. $n=$ even, $n^{\prime}=$ odd: $C P=+1$ state is heavier and decays slower,

3. $n=$ odd, $n^{\prime}=$ even: $C P=+1$ state is lighter and decays faster,

4. $n=$ odd, $n^{\prime}=$ odd: $C P=+1$ state is lighter and decays slower.

Figure 1 illustrates the phase relations in a pictorial way. The choice of $n^{\prime \prime}$ does not alter the conclusion and $n^{\prime \prime}=0$ can be adopted without any loss of generality. In this case, $\left|\mathrm{P}_{+}\right\rangle$is the $C P=+1$ state. 


\subsection{CP Violating Case}

Let us consider the time dependent decay rate for the initial $\mathrm{P}^{0}$ decaying into a $\mathrm{CP}$ eigenstate $\mathrm{f}$, given by $\left|\left\langle f|V| \mathrm{P}^{0}(t)\right\rangle\right|^{2}$, and that for the initial $\overline{\mathrm{P}}^{0}$ decaying into $\mathrm{f}$, given by $\left|\left\langle f|V| \overline{\mathrm{P}}^{0}(t)\right\rangle\right|^{2}$ :

$$
\begin{aligned}
& R_{\mathrm{f}}(t) \propto\left|f_{+}(t)\right|^{2}+\left|\zeta \frac{\bar{A}_{\mathrm{f}}}{A_{\mathrm{f}}}\right|^{2}\left|f_{-}(t)\right|^{2}+2 \Re\left[\zeta \frac{\bar{A}_{\mathrm{f}}}{A_{\mathrm{f}}} f_{+}^{*}(t) f_{-}(t)\right] \\
& \bar{R}_{\mathrm{f}}(t) \propto\left|\frac{\bar{A}_{\mathrm{f}}}{A_{\mathrm{f}}}\right|^{2}\left|f_{+}(t)\right|^{2}+\left|\frac{1}{\zeta}\right|^{2}\left|f_{-}(t)\right|^{2}+\frac{2}{|\zeta|^{2}} \Re\left[\zeta^{*} \frac{\bar{A}_{\mathrm{f}}^{*}}{A_{\mathrm{f}}^{*}} f_{+}^{*}(t) f_{-}(t)\right]
\end{aligned}
$$

where the instantaneous decay amplitudes are denoted by $A_{\mathrm{f}} \equiv\left\langle\mathrm{f}|V| \mathrm{P}^{0}\right\rangle$ etc. and equations 10 and 14 are used.

Since $R_{f}(t)$ and $\bar{R}_{\mathrm{f}}(t)$ describe the CP conjugated processes to each other, any difference between the two is an clear proof of $\mathrm{CP}$ violation. As seen from the first terms of equations 16 and 17, $\mathrm{CP}$ violation is generated if $\left|A_{\mathrm{f}}\right| \neq\left|\bar{A}_{\mathrm{f}}\right|$. This is called $\mathrm{CP}$ violation in the decay amplitudes.

From the second terms of $R_{\mathrm{f}}(t)$ and $\bar{R}_{\mathrm{f}}(t)$, it can be seen that CP violation is generated if $|\zeta| \neq 1$ even if there is no $\mathrm{CP}$ violation in the decay amplitudes. From equations 11 and 15, it is clear that the oscillation rate for $\mathrm{P}^{0} \rightarrow \overline{\mathrm{P}}^{0}$ is different from that for $\overline{\mathrm{P}}^{0} \rightarrow \mathrm{P}^{0}$ if $|\zeta| \neq 1$, thus this is called $\mathbf{C P}$ violation in the oscillation.

The third term can be expanded into

$$
2 \Re\left(\zeta \frac{\bar{A}_{\mathrm{f}}}{A_{\mathrm{f}}}\right) \Re\left[f_{+}^{*}(t) f_{-}(t)\right]-2 \Im\left(\zeta \frac{\bar{A}_{\mathrm{f}}}{A_{\mathrm{f}}}\right) \Im\left[f_{+}^{*}(t) f_{-}(t)\right]
$$

for $R_{\mathrm{f}}(t)$ and

$$
\frac{2}{|\zeta|^{2}} \Re\left(\zeta \frac{\bar{A}_{\mathrm{f}}}{A_{\mathrm{f}}}\right) \Re\left[f_{+}^{*}(t) f_{-}(t)\right]+\frac{2}{|\zeta|^{2}} \Im\left(\zeta \frac{\bar{A}_{\mathrm{f}}}{A_{\mathrm{f}}}\right) \Im\left[f_{+}^{*}(t) f_{-}(t)\right]
$$

for $\bar{R}_{\mathrm{f}}(t)$. If $\mathrm{CP}$ violation in $\mathrm{P}^{0}-\overline{\mathrm{P}}^{0}$ oscillation is absent, the first terms are identical. Even in that case, if

$$
\Im\left(\zeta \frac{\bar{A}_{\mathrm{f}}}{A_{\mathrm{f}}}\right) \neq 0
$$

$\mathrm{CP}$ violation is still present. Since the process involves the decays of $\mathrm{P}^{0}\left(\overline{\mathrm{P}}^{0}\right)$ from the initial $\mathrm{P}^{0}\left(\overline{\mathrm{P}}^{0}\right)$ and decays of the $\overline{\mathrm{P}}^{0}\left(\mathrm{P}^{0}\right)$ oscillated from the initial $\mathrm{P}^{0}\left(\overline{\mathrm{P}}^{0}\right)$ into a common final state, it is referred as $\mathbf{C P}$ violation due to the interplay between the decays and oscillations.

If $\mathrm{CP}$ violation in $\mathrm{P}^{0}-\overline{\mathrm{P}}^{0}$ oscillation is small, i.e. $(|\zeta|-1)^{2}<<1$, we can derive

$$
\left|\sin \left(\varphi_{\Gamma}-\varphi_{M}\right)\right|<<1
$$


from equation 12, where $\varphi_{\Gamma}=\arg \Gamma_{12}$ and $\varphi_{M}=\arg M_{12}$ as already defined. By introducing $\left|\Delta_{\Gamma / M}\right|<<1$ as

$$
\varphi_{\Gamma}-\varphi_{M}=n \pi-\Delta_{\Gamma / M}
$$

where $n$ is an integer number, the following two approximations are possible:

a) $\varphi_{\Gamma}=\arg \Gamma_{12}$ base

$$
\begin{aligned}
\zeta & \approx\left\{1-\frac{2\left|M_{12}\right|\left|\Gamma_{12}\right| \Delta_{\Gamma / M}}{4\left|M_{12}\right|^{2}+\left|\Gamma_{12}\right|^{2}}\left[(-1)^{n+1}+i \frac{2\left|M_{12}\right|}{\left|\Gamma_{12}\right|}\right]\right\} e^{-i \varphi_{\Gamma}} \\
m_{ \pm} & =M \pm(-1)^{n}\left|M_{12}\right| \\
\Gamma_{ \pm} & =\Gamma \pm\left|\Gamma_{12}\right|
\end{aligned}
$$

b) $\varphi_{M}=\arg M_{12}$ base

$$
\begin{aligned}
\zeta & \approx\left\{1+\frac{2\left|M_{12}\right|\left|\Gamma_{12}\right| \Delta_{\Gamma / M}}{4\left|M_{12}\right|^{2}+\left|\Gamma_{12}\right|^{2}}\left[(-1)^{n}+i \frac{\left|\Gamma_{12}\right|}{2\left|M_{12}\right|}\right]\right\} e^{-i \varphi_{M}} \\
m_{ \pm} & =M \pm\left|M_{12}\right| \\
\Gamma_{ \pm} & =\Gamma \pm(-1)^{n}\left|\Gamma_{12}\right| .
\end{aligned}
$$

\section{Neutral Kaon System}

\subsection{Adaptation of Formalism}

Now we adapt the above developed formalism to the neutral kaon system. As described later, observed $\mathrm{CP}$ violation in the $\mathrm{K}^{0}-\overline{\mathrm{K}}^{0}$ oscillation is very small. The two mass eigenstates are called $\mathrm{K}_{\mathrm{S}}$ and $\mathrm{K}_{\mathrm{L}}$ with corresponding masses and decay widths referred to as $m_{\mathrm{S}}, m_{\mathrm{L}}, \Gamma_{\mathrm{S}}$ and $\Gamma_{\mathrm{L}}$ respectively and they are known to be $m_{\mathrm{S}}<m_{\mathrm{L}}$ and $\Gamma_{\mathrm{S}}>\Gamma_{\mathrm{L}}$. Therefore, $M_{12}$ and $\Gamma_{12}$ is almost antiparallel to each other, thus $n=1$ in equation 18 .

Since the kaon decay properties are experimentally well measured, enough information is available to calculate $\Gamma_{12}$ from the data, as described in Section 3.5. We therefore adopt the $\varphi_{\Gamma}$ base given in the previous section.

It follows that

$$
\zeta=(1-2 \epsilon) e^{-i \varphi_{\Gamma}}
$$

where the small parameter $\epsilon$ is given by

$$
\epsilon=\frac{\left|M_{12}\right|\left|\Gamma_{12}\right| \sin \left(\varphi_{\Gamma}-\varphi_{M}\right)}{4\left|M_{12}\right|^{2}+\left|\Gamma_{12}\right|^{2}}\left(1+i \frac{2\left|M_{12}\right|}{\left|\Gamma_{12}\right|}\right) .
$$


and

$$
\begin{aligned}
& \left|\mathrm{K}_{\mathrm{S}}\right\rangle=\frac{1}{\sqrt{1+|\epsilon|^{2}}}\left[\left|\mathrm{~K}^{0}\right\rangle+(1-2 \epsilon) e^{-i \varphi_{\Gamma}}\left|\overline{\mathrm{K}}^{0}\right\rangle\right] \\
& \left|\mathrm{K}_{\mathrm{L}}\right\rangle=\frac{1}{\sqrt{1+|\epsilon|^{2}}}\left[\left|\mathrm{~K}^{0}\right\rangle-(1-2 \epsilon) e^{-i \varphi_{\Gamma}}\left|\overline{\mathrm{K}}^{0}\right\rangle\right] .
\end{aligned}
$$

From the measured lifetimes 12 ,

$$
\tau_{\mathrm{s}} \equiv \frac{1}{\Gamma_{\mathrm{S}}}=(0.8934 \pm 0.0008) \times 10^{-10} \mathrm{~S}
$$

and

$$
\tau_{\mathrm{L}} \equiv \frac{1}{\Gamma_{\mathrm{L}}}=(5.17 \pm 0.04) \times 10^{-8} \mathrm{~S}
$$

i.e.

$$
\Delta \Gamma=\Gamma_{\mathrm{S}}-\Gamma_{\mathrm{L}}=(1.1174 \pm 0.0010) \times 10^{10} \mathrm{~s}^{-1}
$$

and the mass difference,

$$
\Delta m \equiv m_{\mathrm{L}}-m_{\mathrm{S}}=(0.5301 \pm 0.0014) \times 10^{10} \hbar_{\mathrm{s}^{-1}}
$$

we obtain,

$$
\frac{\left|M_{12}\right|\left|\Gamma_{12}\right|}{4\left|M_{12}\right|^{2}+\left|\Gamma_{12}\right|^{2}}=0.24966 \pm 0.00004
$$

and

$$
\frac{2\left|M_{12}\right|}{\left|\Gamma_{12}\right|}=0.9488 \pm 0.0026
$$

Since the lifetime of $\mathrm{K}_{\mathrm{L}}$ is much longer than that of $\mathrm{K}_{\mathrm{S}}$, it is possible to produce a $K_{L}$ beam. Therefore, many kaon experiments have been done using $K_{L}$ beams.

\subsection{CP Violation in Oscillations}

The CPLEAR experiment observed CP violation in the $\mathrm{K}^{0}-\overline{\mathrm{K}}^{0}$ oscillation by measuring the difference in the oscillation rates between $\overline{\mathrm{K}}^{0} \rightarrow \mathrm{K}^{0}$ and $\mathrm{K}^{0} \rightarrow \overline{\mathrm{K}}^{0}$. The initial neutral kaons were produced by $\mathrm{p} \overline{\mathrm{p}}$ annihilations: $\mathrm{p} \overline{\mathrm{p}} \rightarrow \mathrm{K}^{0} \mathrm{~K}^{-} \pi^{+}$and $\rightarrow \overline{\mathrm{K}}^{0} \mathrm{~K}^{+} \pi^{-}$, where the initial flavour can be defined by the charge sign of the accompanying kaon. Semileptonic decays were used in order to determine the flavour at the moment of the decay. Since the $\mathrm{K}^{0}$ contains an $\overline{\mathrm{S}}$-quark (and $\overline{\mathrm{K}}^{0}$ an s-quark), $\mathrm{K}^{0}\left(\overline{\mathrm{K}}^{0}\right)$ can decay only into $\mathrm{e}^{+} \pi^{-} \nu\left(\mathrm{e}^{-} \pi^{+} \bar{\nu}\right)$ instantaneously. Therefore, the initial $\mathrm{K}^{0}\left(\overline{\mathrm{K}}^{0}\right)$ can produce the 


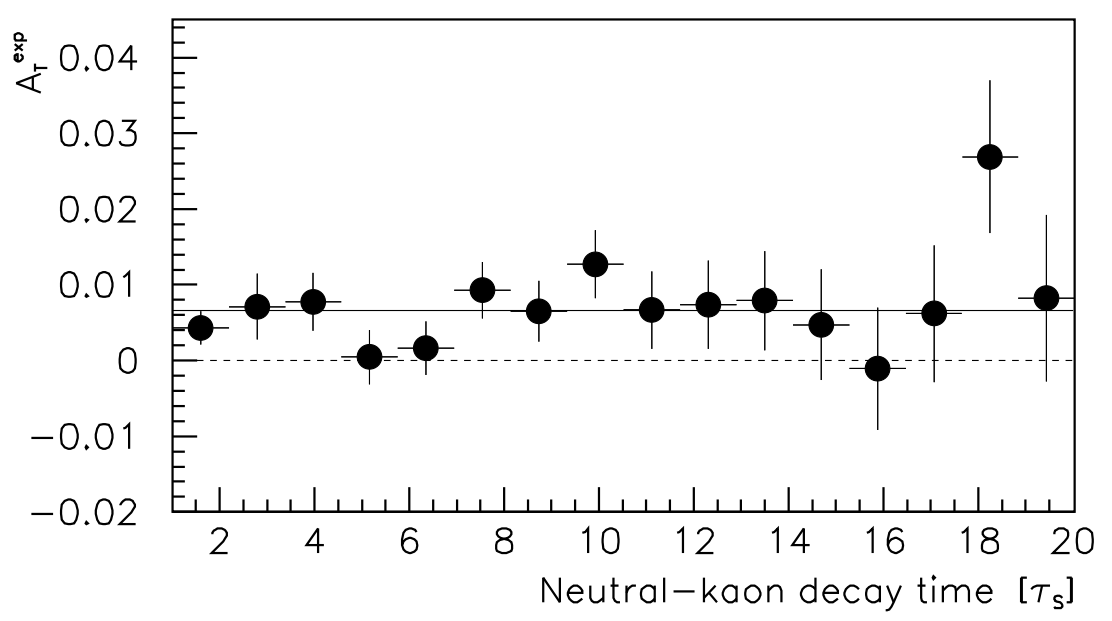

Figure 2: Measured rate asymmetry between the initial $\overline{\mathrm{K}}^{0}$ decaying into $\mathrm{e}^{+} \pi^{-} \nu$ and the initial $\mathrm{K}^{0}$ decaying into $\mathrm{e}^{-} \pi^{+} \bar{\nu}$ as a function of the decay time in units of $\tau_{\mathrm{S}}$ by the CPLEAR experiment. The solid line is obtained by fitting a constant value.

final state $\mathrm{e}^{-} \pi^{+} \bar{\nu}\left(\mathrm{e}^{+} \pi^{-} \nu\right)$ only through the $\mathrm{K}^{0} \rightarrow \overline{\mathrm{K}}^{0}\left(\overline{\mathrm{K}}^{0} \rightarrow \mathrm{K}^{0}\right)$ oscillation. From the two measured time dependent decay rates, $R_{\mathrm{e}^{-}}(t)$ and $\bar{R}_{\mathrm{e}^{+}}(t)$, an asymmetry

$$
A_{\mathrm{T}}(t)=\frac{\bar{R}_{\mathrm{e}^{+}}(t)-R_{\mathrm{e}^{-}}(t)}{\bar{R}_{\mathrm{e}^{+}}(t)+R_{\mathrm{e}^{-}}(t)}
$$

is constructed as shown in Figure 2. Using equations 10, 14 and 20, it follows that

$$
A_{\mathrm{T}}(t)=\frac{1-|\zeta|^{4}}{1+|\zeta|^{4}}=4 \Re \epsilon
$$

and from the measured $A_{\mathrm{T}}(t)=(6.6 \pm 1.6) \times 10^{-3}$ 13n,

$$
|\zeta|=0.9967 \pm 0.0008 \neq 1
$$

is obtained exhibiting a clear sign of $\mathrm{CP}$ violation and $\mathrm{T}$ violation in the $\mathrm{K}^{0}-\overline{\mathrm{K}}^{0}$ oscillation.

The parameter $|\zeta|$ can also be measured from the semileptonic branching fractions of $\mathrm{K}_{\mathrm{L}}$ by the lepton sign asymmetry: using equations 22 and 20, we obtain [12]

$$
\begin{aligned}
\delta_{\ell} & \equiv \frac{B\left(\mathrm{~K}_{\mathrm{L}} \rightarrow \ell^{+} \pi^{-} \nu\right)-B\left(\mathrm{~K}_{\mathrm{L}} \rightarrow \ell^{-} \pi^{+} \bar{\nu}\right)}{B\left(\mathrm{~K}_{\mathrm{L}} \rightarrow \ell^{+} \pi^{-} \nu\right)+B\left(\mathrm{~K}_{\mathrm{L}} \rightarrow \ell^{-} \pi^{+} \bar{\nu}\right)} \\
& =\frac{1-|\zeta|^{2}}{1+|\zeta|^{2}}=2 \Re \epsilon \\
& =(3.27 \pm 0.12) \times 10^{-3}
\end{aligned}
$$


where $\ell$ can be e or $\mu$ and $B$ stands for a branching fraction.

Using all the measurements, we obtain

$$
\Re \epsilon=(1.64 \pm 0.06) \times 10^{-3}
$$

and

$$
\arg \epsilon=(43.50 \pm 0.08)^{\circ}
$$

\subsection{CP Violation due to Decays and Oscillations}

Since the two-pion final state is a $\mathrm{CP}$ eigenstate with $C P=+1, \mathrm{~K}_{\mathrm{L}}$ decaying into $\pi^{+} \pi^{-}$is a CP violating decay. This was indeed the first observed sign of CP violation. A commonly used CP violation parameter $\eta_{+-}$is defined as

$$
\eta_{+-} \equiv \frac{\left\langle\pi^{+} \pi^{-}|V| \mathrm{K}_{\mathrm{L}}\right\rangle}{\left\langle\pi^{+} \pi^{-}|V| \mathrm{K}_{\mathrm{S}}\right\rangle}=\frac{1-\zeta \frac{\bar{A}_{+-}}{A_{+-}}}{1+\zeta \frac{\bar{A}_{+-}}{A_{+-}}}
$$

where equations 21, 22 are used and $A_{+-}$and $\bar{A}_{+-}$denote the $\mathrm{K}^{0}$ and $\overline{\mathrm{K}}^{0} \rightarrow \pi^{+} \pi^{-}$ decay amplitudes respectively.

The parameter $\eta_{+-}$can be measured from the time dependent decay rates for the initial $\mathrm{K}^{0}$ and $\overline{\mathrm{K}}^{0}$ into $\pi^{+} \pi^{-}$. From equations 11 and 15 , the two rates are given by

$$
R_{+-}(t) \propto \frac{1}{2} e^{-\Gamma_{\mathrm{S}} t}+\left|\eta_{+-}\right|^{2} e^{-\Gamma_{\mathrm{L}} t}+2\left|\eta_{+-}\right| e^{-\hat{\Gamma} t} \cos \left(\Delta m t-\phi_{+-}\right)
$$

and

$$
\bar{R}_{+-}(t) \propto \frac{1+4 \Re \epsilon}{2}\left[e^{-\Gamma_{\mathrm{S}} t}+\left|\eta_{+-}\right|^{2} e^{-\Gamma_{\mathrm{L}} t}-2\left|\eta_{+-}\right| e^{-\hat{\Gamma} t} \cos \left(\Delta m t-\phi_{+-}\right)\right]
$$

where $\phi_{+-}$is the phase of $\eta_{+-}$and $\hat{\Gamma}$ is the $\mathrm{K}_{\mathrm{S}}-\mathrm{K}_{\mathrm{L}}$ average decay width. The second term is $\mathrm{CP}$ violating $\mathrm{K}_{\mathrm{L}}$ decays and the third term is due to the interference between the $\mathrm{K}_{\mathrm{S}}$ decay and $\mathrm{CP}$ violating $\mathrm{K}_{\mathrm{L}}$ decay amplitudes. Figure 3 shows [14] the measured $R_{+-}(t)$ and $R_{+-}(t)$ together with the CP asymmetry defined as

$$
A_{+-}(t)=\frac{\bar{R}_{+-}(t)-R_{+-}(t)}{\bar{R}_{+-}(t)+R_{+-}(t)}
$$

where the interference term is well isolated. At around $t=10 \tau_{\mathrm{S}}$, the $\mathrm{K}_{\mathrm{S}}$ decay rate is reduced to the level of the $\mathrm{CP}$ violating $\mathrm{K}_{\mathrm{L}}$ decay rate, thus the asymmetry becomes very large. 

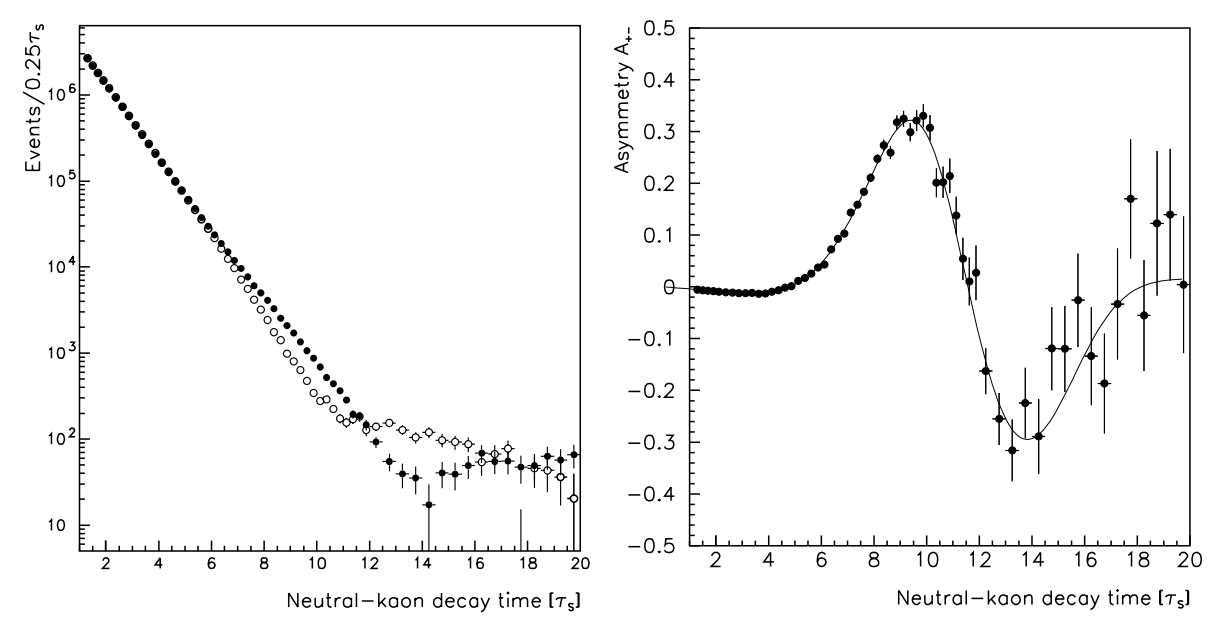

Figure 3: The time dependent rate distributions for the initial $\overline{\mathrm{K}}^{0}$ (solid circles) and $\mathrm{K}^{0}$ (open circles) decaying into $\pi^{+} \pi^{-}$as a function of the decay time in units of $\tau_{\mathrm{S}}$ obtained by the CPLEAR experiment. The rate asymmetry is also shown.

This direct comparison between the two $\mathrm{CP}$ conjugated processes illustrates another straightforward demonstration of $\mathrm{CP}$ violation in the neutral kaon system. From the asymmetry, the value of $\eta_{+-}$is measured to be [14]

$$
\left|\eta_{+-}\right|=(2.264 \pm 0.035) \times 10^{-3}, \phi_{+-}=(43.19 \pm 0.60)^{\circ}
$$

which leads to

$$
\Im\left(\zeta \frac{\bar{A}_{+-}}{A_{+-}}\right)=-(3.099 \pm 0.048) \times 10^{-3}
$$

exhibiting that $\mathrm{CP}$ violation due to the interference between the decay and oscillation is present.

\subsection{CP Violation in Decays}

The two-pion final state can be in a total isospin state of $I=0$ or $I=2$. The $I=1$ state is not allowed due to Bose statistics. Using the isospin decomposition, we can derive the $\mathrm{K}^{0}$ and $\overline{\mathrm{K}}^{0}$ decay amplitudes to $\pi^{+} \pi^{-}$to be

$$
A_{+-}=\sqrt{\frac{2}{3}}\left\langle 2 \pi(I=0)|V| \mathrm{K}^{0}\right\rangle+\sqrt{\frac{1}{3}}\left\langle 2 \pi(I=2)|V| \mathrm{K}^{0}\right\rangle
$$

and

$$
\bar{A}_{+-}=\sqrt{\frac{2}{3}}\left\langle 2 \pi(I=0)|V| \overline{\mathrm{K}}^{0}\right\rangle+\sqrt{\frac{1}{3}}\left\langle 2 \pi(I=2)|V| \overline{\mathrm{K}}^{0}\right\rangle .
$$


Using CPT symmetry and the S-matrix, the $\mathrm{K}^{0}$ and $\overline{\mathrm{K}}^{0}$ decay amplitudes can be related and it follows that

$$
\begin{aligned}
& A_{+-}=\sqrt{\frac{2}{3}} a_{0} e^{i \delta_{0}}+\sqrt{\frac{1}{3}} a_{2} e^{i \delta_{2}} \\
& \bar{A}_{+-}=\sqrt{\frac{2}{3}} a_{0}^{*} e^{i\left(\delta_{0}+\theta_{\mathrm{CP}}-\bar{\theta}_{\mathrm{T}}\right)}+\sqrt{\frac{1}{3}} a_{2}^{*} e^{i\left(\delta_{2}+\theta_{\mathrm{CP}}-\bar{\theta}_{\mathrm{T}}\right)}
\end{aligned}
$$

where $a_{0}$ and $a_{2}$ are the $\mathrm{K}^{0}$ decay amplitudes into $2 \pi(I=0)$ and $2 \pi(I=2)$ states due to the short-range weak interactions and $\delta_{0}$ and $\delta_{2}$ are the $\pi-\pi$ scattering phase shifts for the $I=0$ and $I=2$ two-pion configuration at $\sqrt{s}=m_{\mathrm{K}}$ respectively. It is important to note that the two-pion scattering is totally dominated by the elastic scattering at the energy scale of the kon mass. Similarly for the $\pi^{0} \pi^{0}$ final state, we have

$$
\begin{aligned}
& A_{00}=-\sqrt{\frac{1}{3}} a_{0} e^{i \delta_{0}}+\sqrt{\frac{2}{3}} a_{2} e^{i \delta_{2}} \\
& \bar{A}_{00}=-\sqrt{\frac{1}{3}} a_{0}^{*} e^{i\left(\delta_{0}+\theta_{\mathrm{CP}}-\bar{\theta}_{\mathrm{T}}\right)}+\sqrt{\frac{2}{3}} a_{2}^{*} e^{i\left(\delta_{2}+\theta_{\mathrm{CP}}-\bar{\theta}_{\mathrm{T}}\right)} .
\end{aligned}
$$

As seen from the amplitudes, $B\left(\mathrm{~K}_{\mathrm{S}} \rightarrow \pi^{0} \pi^{0}\right) / B\left(\mathrm{~K}_{\mathrm{S}} \rightarrow \pi^{+} \pi^{-}\right)$would be 0.5 if $a_{2}=0$. Since the measured ratio is $\sim 0.46$ [14], we can conclude that $\left|a_{2} / a_{0}\right|<<1$. It follows that

$$
\frac{\bar{A}_{+-}}{A_{+-}}=\left(1-2 \epsilon^{\prime}\right) e^{-i\left(2 \varphi_{0}+\bar{\theta}_{\mathrm{T}}-\theta_{\mathrm{CP}}\right)}
$$

where the parameter $\epsilon^{\prime}$ is given by

$$
\epsilon^{\prime}=\frac{1}{\sqrt{2}}\left|\frac{a_{2}}{a_{0}}\right| \sin \left(\varphi_{2}-\varphi_{0}\right) e^{i\left(\pi / 2+\delta_{2}-\delta_{0}\right)}
$$

and $\varphi_{0,2}=\arg a_{0,2}$.

As seen from equation 24, $\mathrm{CP}$ violation in the decay amplitude, $\left|A_{+-}\right| \neq\left|\bar{A}_{+-}\right|$, is present if $\Re \epsilon^{\prime} \neq 0$. From equation 25, this is possible only if

$$
\sin \left(\varphi_{2}-\varphi_{0}\right) \neq 0 \text { and } \sin \left(\delta_{2}-\delta_{0}\right) \neq 0 \text {. }
$$

i.e. both the weak and strong phases have to be different for the $I=0$ and $I=$ 2 decay amplitudes. More generally, there must be two processes leading to the identical final state and both the strong and the weak phases must be different between the two processes in order to generate $\mathrm{CP}$ violation in the decay amplitudes. It should be noted that from the measured $\pi-\pi$ scattering phase shift values, we have 15

$$
\arg \epsilon^{\prime}=(43 \pm 6)^{\circ}
$$


Using equations 20 and 24, it follows that

$$
\begin{aligned}
\zeta \frac{\bar{A}_{+-}}{A_{+-}} & =\left(1-2 \epsilon-2 \epsilon^{\prime}\right) e^{-i\left(\varphi_{\Gamma}+2 \varphi_{0}+\bar{\theta}_{\mathrm{T}}-\theta_{\mathrm{CP}}\right)} \\
& \approx 1-2\left(\epsilon+\epsilon^{\prime}\right)-i\left(\varphi_{\Gamma}+2 \varphi_{0}+\bar{\theta}_{\mathrm{T}}-\theta_{\mathrm{CP}}\right)
\end{aligned}
$$

where the approximation is made assuming that the phase difference between $\Gamma_{12}$ and $A_{0} \bar{A}_{0}$ is small, which will be justified later. From equation [23, $\eta_{+-}$can be derived to be

$$
\eta_{+-}=\epsilon+i\left(\varphi_{\Gamma}+2 \varphi_{0}+\bar{\theta}_{\mathrm{T}}-\theta_{\mathrm{CP}}\right)+\epsilon^{\prime}
$$

Similarly the CP violation parameter for the $\pi^{0} \pi^{0}$ decay channel, $\eta_{00}$, is given by

$$
\eta_{00}=\epsilon+i\left(\varphi_{\Gamma}+2 \varphi_{0}+\bar{\theta}_{\mathrm{T}}-\theta_{\mathrm{CP}}\right)-2 \epsilon^{\prime}
$$

Thus, we expect CP violation parameters to be different between the $\pi^{+} \pi^{-}$and $\pi^{0} \pi^{0}$ decay modes if $\epsilon^{\prime} \neq 0$. It has been shown by four recent experiments, NA31 [16], E731 [17], KTeV [18] and NA48 [19],

$$
\left|\frac{\eta_{+-}}{\eta_{00}}\right|^{2}=1.0127 \pm 0.0028
$$

i.e. $\mathrm{CP}$ violation in the decay amplitude is present in the neutral kaon system. If we neglect $\left(\varphi_{\Gamma}+2 \varphi_{0}+\bar{\theta}_{\mathrm{T}}-\theta_{\mathrm{CP}}\right)$, it follows that

$$
\Re\left(\frac{\epsilon^{\prime}}{\epsilon}\right)=\frac{1}{6}\left(\left|\frac{\eta_{+-}}{\eta_{00}}\right|^{2}-1\right) .
$$

\subsection{Phase of Decay Matrix}

As seen from equation 5, evaluation of $\Gamma_{12}$ involves the decay final states which are common to $\mathrm{K}^{0}$ and $\overline{\mathrm{K}}^{0}$, which are $2 \pi(I=0), 2 \pi(I=2), 3 \pi(I=1), 3 \pi(I=2)$ and $3 \pi(I=3)$ states:

$$
\Gamma_{12} \approx \sum_{I=0,2} A_{2 \pi(I)}^{*} \bar{A}_{2 \pi(I)}+\sum_{I=1,2,3} A_{3 \pi(I)}^{*} \bar{A}_{3 \pi(I)}
$$

The contribution from the decay amplitude to the $2 \pi(I=2)$ state is suppressed by the $\Delta I=1 / 2$ rule and the small measured value of $\epsilon^{\prime}$. The contribution from the three-pion decay amplitudes are suppressed by $\Gamma_{\mathrm{L}} / \Gamma_{\mathrm{S}}$ and the measured upper limits for the $\mathrm{CP}$ violation parameter for the $\pi^{+} \pi^{-} \pi^{0}$ and $\pi^{0} \pi^{0} \pi^{0}$ final states. In 
conclusion, the phase of $\Gamma_{12}$ is essentially given by the phase of the $A_{0}$ amplitude, and it can be expressed as

$$
\varphi_{\Gamma} \approx \arg A_{0}^{*} \bar{A}_{0}=-2 \varphi_{0}-\bar{\theta}_{\mathrm{T}}+\theta_{\mathrm{CP}}
$$

so that

$$
\left|\varphi_{\Gamma}+2 \varphi_{0}+\bar{\theta}_{\mathrm{T}}-\theta_{\mathrm{CP}}\right|<O\left(10^{-5}\right) \text {. }
$$

Thus $\left|\varphi_{\Gamma}+2 \varphi_{0}+\bar{\theta}_{\mathrm{T}}-\theta_{\mathrm{CP}}\right|<<|\epsilon|$, justifying the approximations made before.

\subsection{The Standard Model Description}

In the framework of the Standard Model [20], the short range contribution to $\mathrm{K}^{0}-\overline{\mathrm{K}}^{0}$ oscillation is obtained from the box diagrams (Figure 4 ) to be

$$
M_{12}^{\text {box }}=-\frac{G_{\mathrm{F}}^{2} f_{\mathrm{K}}^{2} B_{\mathrm{K}} m_{\mathrm{K}} m_{\mathrm{W}}^{2}}{12 \pi^{2}}\left[\eta_{1} \sigma_{\mathrm{c}}^{2} S\left(x_{\mathrm{c}}\right)+2 \eta_{2} \sigma_{\mathrm{c}} \sigma_{\mathrm{t}} E\left(x_{\mathrm{c}}, x_{\mathrm{t}}\right)+\eta_{3} \sigma_{\mathrm{t}}^{2} S\left(x_{\mathrm{t}}\right)\right]
$$

where $G_{\mathrm{F}}$ is the Fermi constant, $f_{\mathrm{K}}, B_{\mathrm{K}}$ and $m_{\mathrm{K}}$ are the decay constant, $B$ parameter and mass for the K-meson respectively and $m_{\mathrm{W}}$ is the mass of the $\mathrm{W}$-boson. The QCD correction factors are denoted by $\eta_{1}=1.38 \pm 0.20, \eta_{2}=0.57 \pm 0.01$ and $\eta_{3}=0.47 \pm 0.04$ and $S$ and $E$ are known functions of the mass ratios, $x_{\mathrm{i}}=m_{\mathrm{i}}^{2} / m_{\mathrm{W}}^{2}$ for top $(\mathrm{i}=\mathrm{t})$ and charm $(\mathrm{i}=\mathrm{c})$. Note that

$$
S\left(x_{\mathrm{c}}\right) \approx 2.4 \times 10^{-4}, S\left(x_{\mathrm{t}}\right) \approx 2.6, E\left(x_{\mathrm{c}}, x_{\mathrm{c}}\right) \approx 2.2 \times 10^{-3}
$$

for $m_{\mathrm{c}}=1.25 \mathrm{GeV} / c, m_{\mathrm{t}}=174 \mathrm{GeV} / c^{2}$ and $m_{\mathrm{W}}=80 \mathrm{GeV} / c$ [12]. The parameters $\sigma_{\mathrm{c}}$ and $\sigma_{\mathrm{t}}$ are the combination of the elements of the Cabibbo-Kobayashi-Maskawa quark mixing matrix (CKM-matrix),

$$
V_{\mathrm{CKM}}=\left(\begin{array}{ccc}
V_{\mathrm{ud}} & V_{\mathrm{us}} & V_{\mathrm{ub}} \\
V_{\mathrm{cd}} & V_{\mathrm{cs}} & V_{\mathrm{cb}} \\
V_{\mathrm{td}} & V_{\mathrm{ts}} & V_{\mathrm{tb}}
\end{array}\right)
$$

$\sigma_{\mathrm{c}}=V_{\mathrm{cs}} V_{\mathrm{cd}}{ }^{*}$ and $\sigma_{\mathrm{t}}=V_{\mathrm{ts}} V_{\mathrm{td}}{ }^{*}$. We adopt the following approximation of the CKM matrix using the parameters introduced by Wolfenstein [21]:

$$
V_{\mathrm{CKM}} \approx\left(\begin{array}{ccc}
1-\lambda^{2} / 2 & \lambda & A \lambda^{3}(\rho-i \eta) \\
-\lambda-i A^{2} \lambda^{5} \eta & 1-\lambda^{2} / 2 & A \lambda^{2} \\
A \lambda^{3}(1-\tilde{\rho}-i \tilde{\eta}) & -A \lambda^{2}-i A \lambda^{4} \eta & 1
\end{array}\right)
$$

where where $\tilde{\rho}=\rho\left(1-\lambda^{2} / 2\right)$ and $\tilde{\eta}=\eta\left(1-\lambda^{2} / 2\right)$. The parameter $\lambda$ is known from the light hadron decays to be $0.221 \pm 0.002$. From the B-meson decays, $\left|V_{\mathrm{cb}}\right|=0.0402 \pm$ 

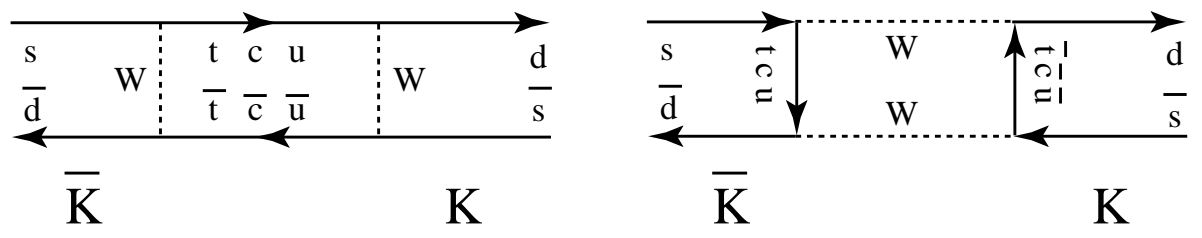

Figure 4: The box diagrams contributing to the $\mathrm{K}^{0}-\overline{\mathrm{K}}^{0}$ oscillations.

0.0019 and $\left|V_{\mathrm{ub}} / V_{\mathrm{cb}}\right|=0.090 \pm 0.025$ are measured [12], giving $A=0.823 \pm 0.042$ and $\sqrt{\rho^{2}+\eta^{2}}=0.41 \pm 0.11$. The $B$-parameter takes in account the difference between $\left\langle 0 \mid H_{\mathrm{W}} \mathrm{K}^{ \pm}\right\rangle$and $\left\langle f\left|H_{\mathrm{W}}\right| \mathrm{K}^{0}\right\rangle$ where $\langle 0|$ is the hadronic vacuum state and $\langle f|$ is the common quark states between $\mathrm{K}^{0}$ and $\overline{\mathrm{K}}^{0}$. The theoretical evaluations for this value vary between 0.5 and 1 .

In addition to $M_{12}^{\text {box }}$, there are large contributions from long range interactions $M_{12}^{\mathrm{LR}}$, which are difficult to evaluate. Therefore, theoretical predication for $M_{12}=$ $M_{12}^{\text {box }}+M_{12}^{\mathrm{LR}}$ cannot be given. The long range interaction involves only the light flavours and its contribution to $M_{12}$ is real in the CKM phase convention; the imaginary part of $M_{12}$ is generated only by the box diagram. Therefore we can derive

$$
\sin \left(\varphi_{M}\right)=\frac{\Im M_{12}}{\left|M_{12}\right|}=\frac{2 \Im M_{12}^{\mathrm{box}}}{\Delta m}
$$

In the CKM phase convention, $\Gamma_{12}$ can be approximated as real. Therefore, it follows that

$$
\Re \epsilon=-\frac{\Im M_{12}^{\mathrm{box}}}{2 \Delta m} .
$$

Although there are considerable uncertainties to evaluate numerically this expression, the currently allowed range of the Wolfenstein parameters, $\lambda, A, \rho$ and $\eta$ gives a consistent value of $\Re \epsilon$ with the experimentally measured value.

Prediction of $\epsilon^{\prime}$ requires an accurate evaluation of the phase difference between $a_{0}$ and $a_{2}$. For the $a_{0}$ amplitudes, the tree, the gluonic penguin and the electroweak penguin diagrams contribute. Only the tree and electroweak penguin diagrams make contributions to the $a_{2}$ decay amplitude. All the penguin diagrams are shown in Figure 5. Not only the short range interactions, but also the hadronic matrix elements with long range interactions have to be evaluated in the calculations. This makes the numerical determination of $\epsilon^{\prime}$ very difficult. Within the theoretical uncertainties, values of $\epsilon^{\prime}$ calculated with the currently allowed range of $\lambda, A, \rho$ and $\eta$ are consistent with the data. 

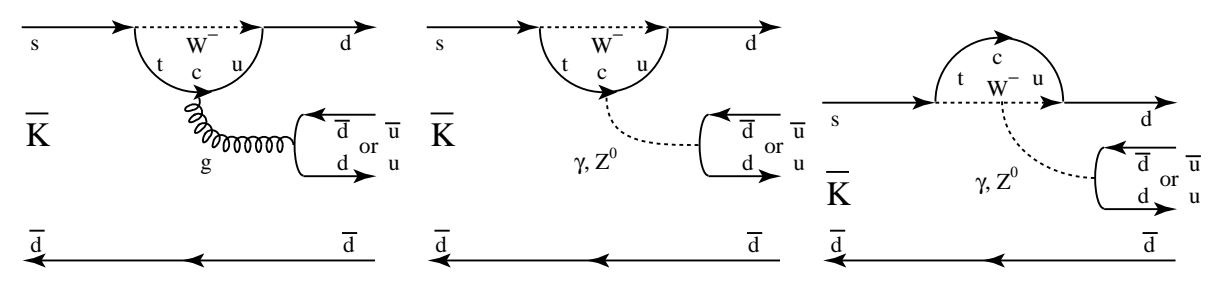

Figure 5: Gluonic and electromagnetic penguins contributing to the $\overline{\mathrm{K}}^{0} \rightarrow 2 \pi$ decays.

\subsection{CP Violation in Rare Decays}

Experimental detection of $\mathrm{K}_{\mathrm{L}} \rightarrow \pi^{0} \nu \bar{\nu}$ is clearly very challenging. The final state is a CP eigenstate with $C P=+1$. Therefore, observation of this decay is a sign of $\mathrm{CP}$ violation. In the Standard Model, the decay is generated by penguin diagrams or box diagrams as shown in Figure 6 .

Since the final state consists with only one hadron, long range strong interactions do not play a role and the decay amplitudes can be denoted as

$$
\begin{aligned}
\left\langle\pi^{0} \nu \bar{\nu}\left|H_{\mathrm{W}}\right| \mathrm{K}^{0}\right\rangle & =a_{\pi^{0} \nu \bar{\nu}} \\
\left\langle\pi^{0} \nu \bar{\nu}\left|H_{\mathrm{W}}\right| \overline{\mathrm{K}}^{0}\right\rangle & =a_{\pi^{0} \nu \bar{\nu}}^{*} e^{i\left(\theta_{\mathrm{CP}}-\bar{\theta}_{\mathrm{T}}\right)} .
\end{aligned}
$$

Unlike for the $\mathrm{K}^{0} \rightarrow 2 \pi$ decays, $\phi_{\pi^{0} \nu \bar{\nu}}=\arg a_{\pi^{0} \nu \bar{\nu}}$ could be very different from $\phi_{0}$, so that we could have a situation

$$
\begin{aligned}
\left|\sin \left(\phi_{\Gamma}+2 \phi_{\pi^{0} \nu \bar{\nu}}+\theta_{\mathrm{CP}}-\bar{\theta}_{\mathrm{T}}\right)\right| & =\left|\sin \left(2 \phi_{\pi^{0} \nu \bar{\nu}}-2 \phi_{0}\right)\right| \\
& >|\epsilon| .
\end{aligned}
$$

The $\mathrm{K}_{\mathrm{L}}$ decay amplitude then becomes

$$
\begin{aligned}
\left\langle\pi^{0} \nu \bar{\nu}\left|H_{\mathrm{W}}\right| \mathrm{K}_{\mathrm{L}}\right\rangle & =\frac{a_{\pi^{0} \nu \bar{\nu}}}{\sqrt{2}}\left[1-(1-2 \epsilon) e^{-i\left(2 \phi_{\pi^{0} \nu \bar{\nu}}-2 \phi_{0}\right)}\right] \\
& \approx \sqrt{2} i\left|a_{\pi^{0} \nu \bar{\nu}}\right| \sin \left(2 \phi_{\pi^{0} \nu \bar{\nu}}-2 \phi_{0}\right) .
\end{aligned}
$$
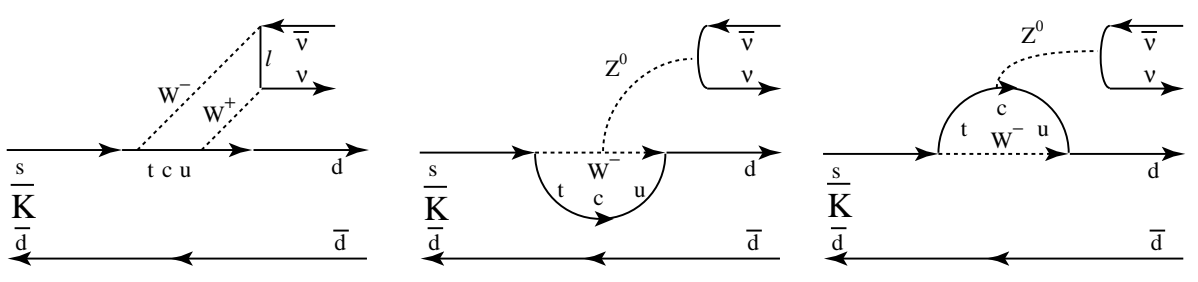

Figure 6: The box and penguin diagrams generating $\overline{\mathrm{K}}^{0} \rightarrow \pi^{0} \nu \bar{\nu}$ decays. 
Using isospin symmetry, the hadronic matrix element of the $\mathrm{K}^{0} \rightarrow \pi^{0} \nu \bar{\nu}$ decay amplitude and that of the $\mathrm{K}^{+} \rightarrow \pi^{+} \mathrm{e}^{+} \nu$ decay amplitudes can be related as

$$
\left\langle\pi^{0}\left|H_{\mathrm{W}}\right| \mathrm{K}^{0}\right\rangle=\left\langle\pi^{0}\left|H_{\mathrm{W}}\right| \mathrm{K}^{+}\right\rangle
$$

This allow us to express the branching fractions for $\mathrm{K}_{\mathrm{L}} \rightarrow \pi^{0} \nu \bar{\nu}$ using the branching fractions for $\mathrm{K}^{+} \rightarrow \pi^{0} \mathrm{e}^{+} \nu$ as [20]

$$
\begin{aligned}
B\left(\mathrm{~K}_{\mathrm{L}} \rightarrow \pi^{0} \nu \bar{\nu}\right) & =\frac{\left|\left\langle\pi^{0} \nu \bar{\nu}\left|H_{\mathrm{W}}\right| \mathrm{K}_{\mathrm{L}}\right\rangle\right|^{2}}{\Gamma_{\mathrm{L}}} \\
& =B\left(\mathrm{~K}^{+} \rightarrow \pi^{0} \mathrm{e}^{+} \nu\right) \frac{\tau_{\mathrm{L}}}{\tau_{+}} \frac{3 \alpha^{2}\left[\Im\left(V_{\mathrm{ts}}^{*} V_{\mathrm{td}}\right) X\left(m_{t}\right)\right]^{2}}{\left|V_{\mathrm{us}}\right|^{2} 2 \pi^{2} \sin ^{4} \Theta_{\mathrm{W}}} \\
& =B\left(\mathrm{~K}^{+} \rightarrow \pi^{0} \mathrm{e}^{+} \nu\right) \frac{\tau_{\mathrm{L}}}{\tau_{+}} \frac{3 \alpha^{2}\left[X\left(m_{t}\right)\right]^{2}}{2 \pi^{2} \sin ^{4} \Theta_{\mathrm{W}}} A^{4} \lambda^{8}\left(1-\lambda^{2} / 2\right)^{2} \eta^{2} \\
& \approx 3 \times 10^{-11}
\end{aligned}
$$

where $X$ is a known function and $\Theta_{\mathrm{W}}$ is the weak mixing angle. Since the hadronic matrix element is taken from the data, the theoretical uncertainties in this determination is very small. Also the imaginary part of the amplitude is dominated by the short range interactions which can be reliably calculated. Therefore, the theoretical prediction can be considered to be clean.

It is interesting to note that the $\mathrm{CP}$ violation parameter

$$
\eta_{\pi^{0} \nu \bar{\nu}}=\frac{\left\langle\pi^{0} \nu \bar{\nu}|V| \mathrm{K}_{\mathrm{L}}\right\rangle}{\left\langle\pi^{0} \nu \bar{\nu}|V| \mathrm{K}_{\mathrm{S}}\right\rangle}
$$

as defined in the $2 \pi$ case has $\left|\eta_{\pi^{0} \nu \bar{\nu}}\right|>>|\epsilon|$, although the both final states have $C P=+1$.

The current experimental measurement for this branching fraction is $<5.9 \times 10^{-7}$ with $90 \%$ confidence by the $\mathrm{KTeV}$ experiment [22], which is still far from the expected number. However, there are several proposals to observe the decays in the near future.

\section{B-meson System}

\subsection{The Standard Model Description}

\subsubsection{Some Elements of The CKM Matrix}

Among the nine elements of the CKM matrix, five of them related to the third generation play important roles in the $\mathrm{B}$ meson system: $V_{\mathrm{td}}, V_{\mathrm{ub}}, V_{\mathrm{ts}}, V_{\mathrm{cb}}$ and $V_{\mathrm{tb}}$. 


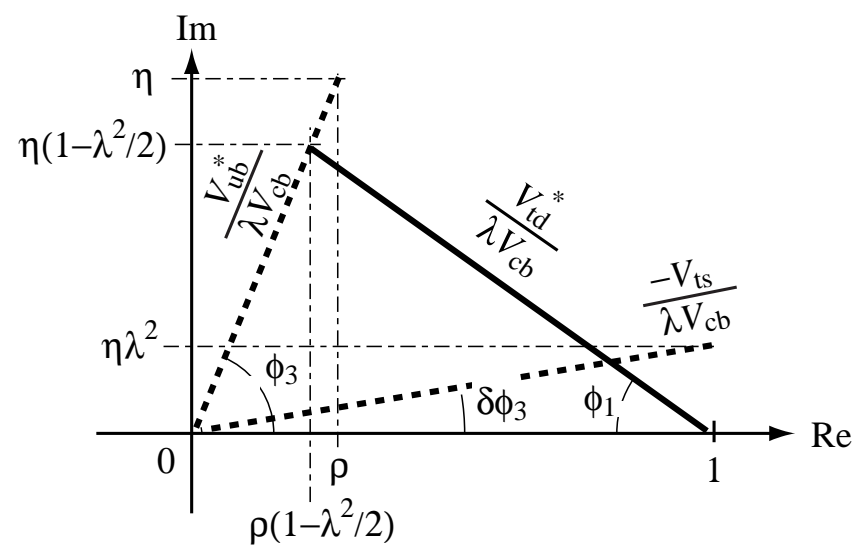

Figure 7: Three elements of the CKM matrix, $V_{\mathrm{td}}, V_{\mathrm{ub}}$, and $V_{\mathrm{ts}}$ and the definitions of $\phi_{1}, \phi_{3}$ and $\delta \phi_{3}$.

In the approximation given in equation 27, the phases of the five elements are given by

$$
\arg V_{\mathrm{td}}=-\phi_{1}, \quad \arg V_{\mathrm{ub}}=-\phi_{3}, \quad \arg V_{\mathrm{ts}}=\delta \phi_{3}+\pi, \quad \arg V_{\mathrm{cb}}=\arg V_{\mathrm{tb}}=0
$$

where

$$
\phi_{1}=\tan ^{-1} \frac{\eta}{1-\rho}, \phi_{3}=\tan ^{-1} \frac{\eta}{\rho}, \delta \phi_{3}=\tan ^{-1} \lambda^{2} \eta .
$$

Figure 7 shows the angles in $\rho$ and $\eta$ planes. Note that $\phi_{1}$ and $\phi_{3}$ are often referred to as $\beta$ and $\gamma$. Clearly $\delta \phi_{3}$ is very small, $\sim 0.02$.

\subsubsection{Oscillation Amplitude}

In the Standard Model, B-B oscillation is totally governed by the short range interactions, i.e. the box diagrams. Furthermore, only the top quark plays a role in the box diagram due to the large top quark mass (see equation 26) and the structure of the CKM matrix;

$$
\frac{\Re\left(V_{\mathrm{td}}^{*} V_{\mathrm{tb}}\right)}{\Re\left(V_{\mathrm{cd}}^{*} V_{\mathrm{cb}}\right)}=(\tilde{\rho}-1) \approx 1, \frac{\Im\left(V_{\mathrm{td}}^{*} V_{\mathrm{tb}}\right)}{\Im\left(V_{\mathrm{cd}}^{*} V_{\mathrm{cb}}\right)} \approx \frac{1}{\lambda^{2}}>>1
$$

as seen from equation 27 .

Therefore, the off diagonal element of the mass matrix, $M_{12}$ is given by 20

$$
M_{12}=-\frac{G_{\mathrm{F}}^{2} f_{\mathrm{B}_{\mathrm{d}}}^{2} B_{\mathrm{B}_{\mathrm{d}}} m_{\mathrm{B}_{\mathrm{d}}} m_{\mathrm{W}}^{2}}{12 \pi^{2}} \eta_{\mathrm{B}_{\mathrm{d}}} S\left(x_{\mathrm{t}}\right)\left(V_{\mathrm{td}}^{*} V_{\mathrm{tb}}\right)^{2} \quad \text { for } \mathrm{B}_{\mathrm{d}}
$$


where $f_{\mathrm{B}_{\mathrm{d}}}, B_{\mathrm{B}_{\mathrm{d}}}$ and $m_{\mathrm{B}_{\mathrm{d}}}$ are the decay constant, B-parameter and the mass of the $\mathrm{B}_{\mathrm{d}}$ meson.

Similarly for the $\mathrm{B}_{\mathrm{s}}$ meson, we obtain

$$
M_{12}=-\frac{G_{\mathrm{F}}^{2} f_{\mathrm{B}_{\mathrm{s}}}^{2} B_{\mathrm{B}_{\mathrm{s}}} m_{\mathrm{B}_{\mathrm{s}}} m_{\mathrm{W}}^{2}}{12 \pi^{2}} \eta_{\mathrm{B}_{\mathrm{s}}} S\left(x_{\mathrm{t}}\right)\left(V_{\mathrm{ts}}^{*} V_{\mathrm{tb}}\right)^{2} \quad \text { for } \mathrm{B}_{\mathrm{s}}
$$

where $f_{\mathrm{B}_{\mathrm{s}}}, B_{\mathrm{B}_{\mathrm{s}}}$ and $m_{\mathrm{B}_{\mathrm{s}}}$ are the decay constant, B-parameter and the mass of the $\mathrm{B}_{\mathrm{s}}$ meson.

The phase of $M_{12}$ is then given by

$$
\arg M_{12}= \begin{cases}\arg \left(V_{\mathrm{td}}^{*} V_{\mathrm{tb}}\right)^{2}+\pi=2 \phi_{1}+\pi & \text { for } \mathrm{B}_{\mathrm{d}} \\ \arg \left(V_{\mathrm{ts}}^{*} V_{\mathrm{tb}}\right)^{2}+\pi=-2 \delta \phi_{3}+\pi & \text { for } \mathrm{B}_{\mathrm{s}} .\end{cases}
$$

The parameter $\Gamma_{12}$ can also be determined by taking the absorptive part of the box diagrams with charm and up quarks in the loops. The phase difference between $M_{12}$ and $\Gamma_{12}$ is given by

$$
\arg M_{12}-\arg \Gamma_{12}=\pi+\frac{8}{3}\left(\frac{m_{\mathrm{c}}}{m_{\mathrm{b}}}\right)^{2} \eta \times\left\{\begin{array}{cl}
\frac{1}{(1-\rho)^{2}+\eta^{2}} & : \mathrm{B}_{\mathrm{d}} \\
\lambda^{2} & : \mathrm{B}_{\mathrm{s}}
\end{array}\right.
$$

i.e. $\sin \left(\arg M_{12}-\arg \Gamma_{12}\right)$ is small for $\mathrm{B}_{\mathrm{d}}$ and very small for $\mathrm{B}_{\mathrm{s}}$. Note that $M_{12}$ and $\Gamma_{12}$ are antiparallel. Therefore, the approximations for $\zeta, m_{ \pm}$and $\Gamma_{ \pm}$given on page 8 are valid with $n=1$. Since we will rely on the Standard Model description of $M_{12}$, and our experimental knowledge of the decay amplitudes is still limited, we adopt b) $\phi_{M}$ base. We refer the mass eigenstate with larger mass as $\mathrm{B}_{\mathrm{h}}$ (B-heavy) and the other $\mathrm{B}_{1}$ (B-light) with their masses and decay width are given by:

$$
m_{\mathrm{h}}=M+\left|M_{12}\right|, \Gamma_{\mathrm{h}}=\Gamma-\left|\Gamma_{12}\right|
$$

and

$$
m_{1}=M-\left|M_{12}\right|, \Gamma_{1}=\Gamma+\left|\Gamma_{12}\right|
$$

respectively, and $B_{h}\left(B_{l}\right)$ corresponds to $P_{+}\left(P_{-}\right)$defined in equation 13 .

For both $\mathrm{B}_{\mathrm{d}}$ and $\mathrm{B}_{\mathrm{s}}$, we can now derive

$$
\frac{\Delta \Gamma}{\Delta m}=\left|\frac{\Gamma_{12}}{M_{12}}\right| \approx \frac{3 \pi m_{\mathrm{b}}^{2}}{2 m_{\mathrm{W}}^{2} S\left(x_{\mathrm{t}}\right)} \approx 5 \times 10^{-3} \quad \text { for } \mathrm{B}_{\mathrm{d}} \text { and } \mathrm{B}_{\mathrm{s}}
$$

for $m_{\mathrm{b}}=4.25 \mathrm{GeV}, m_{\mathrm{W}}=80 \mathrm{GeV}$ and $m_{\mathrm{t}}=174 \mathrm{GeV}$, where $\Delta m$ and $\Delta \Gamma$ are defined as positive:

$$
\Delta m=m_{\mathrm{h}}-m_{\mathrm{l}}, \Delta \Gamma=\Gamma_{\mathrm{l}}-\Gamma_{\mathrm{h}} .
$$


Using the measured values of $\Delta m=(0.464 \pm 0.018) \times 10^{12} \hbar \mathrm{s}^{-1}$ and the average lifetime $\tau=1 / \hat{\Gamma}=(1.54 \pm 0.03) \times 10^{-12} \mathrm{~s}$ for the $\mathrm{B}_{\mathrm{d}}$ mesons, where $\hat{\Gamma}$ is the averaged decay width, it follows that

$$
\frac{\Delta \Gamma}{\hat{\Gamma}} \approx 4 \times 10^{-3} \quad \text { for } \mathrm{B}_{\mathrm{d}}
$$

and $\Delta \Gamma$ can be neglected in the decay time distribution for the $\mathrm{B}_{\mathrm{d}}$ system. For the $\mathrm{B}_{\mathrm{s}}$ mesons, using the measured lifetime $(1.54 \pm 0.07) \times 10^{-12} \mathrm{~s}$, it follows that

$$
\frac{\Delta \Gamma}{\hat{\Gamma}} \approx 0.1 \quad \text { for } \mathrm{B}_{\mathrm{s}}
$$

The effect of $\Delta \Gamma$ is still not large, but can no longer be neglected in the decay time distributions.

The small decay width differences of the $B_{d}$ and $B_{s}$ systems do not allow to separate one mass-eigenstate from the other, which can be done for the kaon system by creating a $\mathrm{K}_{\mathrm{L}}$ beam. Therefore, $\mathrm{CP}$ violation cannot be established by just observing the decays as in the case of $\mathrm{K}_{\mathrm{L}} \rightarrow 2 \pi$. We either have to compare the decay rates of the initial $\mathrm{B}^{0}$ and initial $\overline{\mathrm{B}}^{0}$ states or measure the time dependent decay rates of at least one of the two cases, i.e. either initial $\mathrm{B}^{0}$ or $\overline{\mathrm{B}}^{0}$.

Since $\Delta m=2\left|M_{12}\right|$, one can extract

$$
\left|V_{\mathrm{td}}\right|^{2}=A^{4} \lambda^{6}\left[(1-\tilde{\rho})^{2}+\tilde{\eta}^{2}\right]
$$

i.e. $\rho$ and $\eta$, from the measured $\mathrm{B}^{0}-\overline{\mathrm{B}}^{0}$ oscillation frequency $\Delta m_{\mathrm{d}}$ using equation 28 . However, theoretical uncertainties in calculating the decay constant and B-parameter are considerable and limit the accuracy on the extracted value of $\left|V_{\mathrm{td}}\right|^{2}$. If the $\mathrm{B}_{\mathrm{s}}^{0}-\overline{\mathrm{B}}_{\mathrm{s}}^{0}$ oscillation frequency $\Delta m_{\mathrm{s}}=2\left|M_{12}^{\mathrm{s}}\right|$ is measured, $\left|V_{\mathrm{td}}\right|^{2}$ can be determined with much small uncertainty by using the ratio $\Delta m_{\mathrm{d}} / \Delta m_{\mathrm{s}}$, due to better controlled theoretical errors in $f_{\mathrm{B}_{\mathrm{d}}} / f_{\mathrm{B}_{\mathrm{s}}}$ and $B_{\mathrm{B}_{\mathrm{d}}} / B_{\mathrm{B}_{\mathrm{s}}}$. However, the frequency of the $\mathrm{B}_{\mathrm{s}}^{0}-\overline{\mathrm{B}}_{\mathrm{s}}^{0}$ oscillation is expected to be $>1 / \lambda^{2}=20$ times larger than that of the $\mathrm{B}^{0}-\overline{\mathrm{B}}^{0}$ oscillation and we still have to wait for sometime before it is measured.

Since $\left|M_{12} / \Gamma_{12}\right|<<1, \zeta$ given by equation 19 can be further approximated as

$$
\zeta \approx\left[1-\frac{1}{2} \Im\left(\frac{\Gamma_{12}}{M_{12}}\right)\right] e^{-i \varphi_{M}}
$$

where $\varphi_{M}=\arg M_{12}$ as before. Seen from equation 29 and 30, the approximation $|\zeta| \approx 1$ is accurate to $10^{-3}$ or better.

Similar to the kaon system, CP violation (and T violation) in the oscillation can be measured from the time-dependent rate asymmetry between the initial $\overline{\mathrm{B}}^{0}$ decaying 
into semileptonic final states with $\mathrm{e}^{+}$or $\mu^{+}, \bar{R}_{+}(t)$ and the initial $\mathrm{B}^{0}$ decaying into semileptonic final states with $\mathrm{e}^{-}$or $\mu^{-}, R_{-}(t)$. The asymmetry is given by

$$
\frac{\bar{R}_{+}(t)-R_{-}(t)}{\bar{R}_{+}(t)+R_{-}(t)}=\frac{1-|\zeta|^{4}}{1+|\zeta|^{4}} \approx O\left(10^{-3}\right) \text { for } \mathrm{B}_{\mathrm{d}} \text { and }<<O\left(10^{-3}\right) \text { for } \mathrm{B}_{\mathrm{d}}
$$

which is a very small signal.

From now on, we assume

$$
\zeta=e^{-i \varphi_{M}}
$$

for both $\mathrm{B}_{\mathrm{d}}$ and $\mathrm{B}_{\mathrm{s}}$ and $\Delta \Gamma=0$ for $\mathrm{B}_{\mathrm{d}}$.

In summary, the two mass eigenstates are given by

$$
\begin{aligned}
\left|\mathrm{B}_{\mathrm{h}}\right\rangle & =\frac{1}{\sqrt{2}}\left[|\mathrm{~B}\rangle+e^{-i \varphi_{M}}|\overline{\mathrm{B}}\rangle\right] \\
\left|\mathrm{B}_{\mathrm{l}}\right\rangle & =\frac{1}{\sqrt{2}}\left[|\mathrm{~B}\rangle-e^{-i \varphi_{M}}|\overline{\mathrm{B}}\rangle\right]
\end{aligned}
$$

and

$$
m_{\mathrm{h}}=m_{0}+\left|M_{12}\right|, m_{1}=m_{0}-\left|M_{12}\right|, \Delta m=m_{\mathrm{h}}-m_{1}
$$

for $\mathrm{B}_{\mathrm{d}}$ and $\mathrm{B}_{\mathrm{s}}$. For the decay width, we have

$$
\begin{array}{ll}
\Gamma_{\mathrm{l}}=\Gamma_{\mathrm{h}} & \text { for } \mathrm{B}_{\mathrm{d}} \\
\Gamma_{\mathrm{l}}=\Gamma_{0}+\left|\Gamma_{12}\right|, \Gamma_{\mathrm{h}}=\Gamma_{0}-\left|\Gamma_{12}\right|, \Delta \Gamma=\Gamma_{1}-\Gamma_{\mathrm{h}} & \text { for } \mathrm{B}_{\mathrm{s}}
\end{array}
$$

\subsubsection{Time Dependent Decay Rates}

Since $\Delta \Gamma$ is small in the $\mathrm{B}$ meson system, it is more convenient to derive the time dependent decay rate from the particle-antiparticle base rather than the mass eigenstate base. Using, equations 10 and 14 the time dependent decay rates for the final state $\mathrm{f}$ can be derived as

$$
\begin{aligned}
& R_{\mathrm{f}}(t) \propto \frac{\left|A_{\mathrm{f}}\right|^{2}}{2} e^{-\hat{\Gamma} t}\left[I_{+}(t)+I_{-}(t)\right] \\
& \bar{R}_{\mathrm{f}}(t) \propto \frac{\left|A_{\mathrm{f}}\right|^{2}}{2|\zeta|^{2}} e^{-\hat{\Gamma} t}\left[I_{+}(t)-I_{-}(t)\right]
\end{aligned}
$$

where $\hat{\Gamma}$ is the averaged decay time, $\hat{\Gamma}=\left(\Gamma_{+}+\Gamma_{-}\right) / 2$, and $A_{\mathrm{f}}$ is the instantaneous decay amplitude for the $\mathrm{P}^{0} \rightarrow \mathrm{f}$ decays. The two time dependent functions, $I_{+}(t)$ and $I_{-}(t)$, are given by

$$
\begin{aligned}
& I_{+}(t)=\left(1+\left|L_{\mathrm{f}}\right|^{2}\right) \cosh \frac{\Delta \Gamma}{2} t+2 \Re L_{\mathrm{f}} \sinh \frac{\Delta \Gamma}{2} t \\
& I_{-}(t)=\left(1-\left|L_{\mathrm{f}}\right|^{2}\right) \cos \Delta m t+2 \Im L_{\mathrm{f}} \sin \Delta m t
\end{aligned}
$$


The parameter $L_{\mathrm{f}}$ is given by

$$
L_{\mathrm{f}}=\zeta \frac{\bar{A}_{\mathrm{f}}}{A_{\mathrm{f}}}
$$

where $\bar{A}_{\mathrm{f}}$ is the instantaneous decay amplitude for the $\overline{\mathrm{P}}^{0} \rightarrow \mathrm{f}$ decays.

The time dependent decay rate for the $\mathrm{CP}$ conjugated final states $\mathrm{f}^{\mathrm{CP}}$ are derived to be

$$
\begin{aligned}
& \bar{R}_{\mathrm{fCP}}(t) \propto \frac{\left|\bar{A}_{\mathrm{fCP}}\right|^{2}}{2} e^{-\bar{\Gamma} t}\left[\bar{I}_{+}^{\mathrm{CP}}(t)+\bar{I}_{-}^{\mathrm{CP}}(t)\right] \\
& R_{\mathrm{fCP}}(t) \propto \frac{\left|\bar{A}_{\mathrm{fCP}}\right|^{2}|\zeta|^{2}}{2} e^{-\bar{\Gamma} t}\left[\bar{I}_{+}^{\mathrm{CP}}(t)-\bar{I}_{-}^{\mathrm{CP}}(t)\right]
\end{aligned}
$$

where $\bar{A}_{\mathrm{fCP}}$ is the instantaneous decay amplitude for the $\overline{\mathrm{P}}^{0} \rightarrow \mathrm{f}^{\mathrm{CP}}$ decays. Two time dependent decay rates, $\bar{I}_{+}^{\mathrm{CP}}(t)$ and $\bar{I}_{-}^{\mathrm{CP}}(t)$ are given by

$$
\begin{aligned}
& \bar{I}_{+}^{\mathrm{CP}}(t)=\left(1+\left|L_{\mathrm{f}}^{\mathrm{CP}}\right|^{2}\right) \cosh \frac{\Delta \Gamma}{2} t+2 \Re L_{\mathrm{f}}^{\mathrm{CP}} \sinh \frac{\Delta \Gamma}{2} t \\
& \bar{I}_{-}^{\mathrm{CP}}(t)=\left(1-\left|L_{\mathrm{f}}^{\mathrm{CP}}\right|^{2}\right) \cos \Delta m t+2 \Im L_{\mathrm{f}}^{\mathrm{CP}} \sin \Delta m t
\end{aligned}
$$

where the parameter, $L_{\mathrm{f}}^{\mathrm{CP}}$, is given by

$$
L_{\mathrm{f}}^{\mathrm{CP}}=\frac{1}{\zeta} \frac{A_{\mathrm{f} C \mathrm{P}}}{\bar{A}_{\mathrm{f} \mathrm{CP}}}
$$

and $A_{\mathrm{fCP}}$ is the instantaneous decay amplitude for the $\mathrm{P}^{0} \rightarrow \mathrm{f}^{\mathrm{CP}}$ decays.

The decay rates $R_{f}(t)$ and $\bar{R}_{\mathrm{fCP}}(t)$ are $\mathrm{CP}$ conjugate to each other and so are $\bar{R}_{f}(t)$ and $R_{\mathrm{f}} \mathrm{CP}(t)$. If there exists any difference between the CP conjugated processes, this is a clear sign of $\mathrm{CP}$ violation.

The final state $\mathrm{f}$ can be classified into the following four different cases:

I. Flavour specific final state $\left(A_{\mathrm{f}}=\bar{A}_{\mathrm{f} C \mathrm{P}}=0\right.$ or $\left.A_{\mathrm{fCP}}=\bar{A}_{\mathrm{f}}=0\right)$

II. Flavour non specific final state

II-a. CP eigenstate $\left(A_{\mathrm{f}}=A_{\mathrm{f} C P}\right.$ and $\left.\bar{A}_{\mathrm{f}}=\bar{A}_{\mathrm{fCP}}\right)$

II-b. mixed CP eigenstate $\left(A_{\mathrm{f}}=A_{\mathrm{f} C P}\right.$ and $\left.\bar{A}_{\mathrm{f}}=\bar{A}_{\mathrm{f}}{ }\right)$

II-c. CP non eigenstate 


\subsubsection{CP Violation: Clean Case}

The contribution to the $\mathrm{B}^{0}$ decaying into $\mathrm{J} / \psi \mathrm{K}_{\mathrm{S}}$ is dominated by the tree diagram with $V_{\mathrm{cb}}^{*} V_{\mathrm{cs}}$. Although there exist some contribution from the penguin diagrams, the dominant penguin diagram contribution has the CKM phase $V_{\mathrm{tb}}^{*} V_{\mathrm{ts}}$ which is close to that of the tree diagram (Figure 8). Thus, we can safely assume that there is no CP violation in the decay amplitude and the ratio of the $\overline{\mathrm{B}}^{0}$ and $\mathrm{B}^{0}$ decay amplitudes is given only by the CKM part. By noting that $C P\left(\mathrm{~J} / \psi \mathrm{K}_{\mathrm{S}}\right)=-1$ we obtain

$$
\frac{A\left(\overline{\mathrm{B}}^{0} \rightarrow \mathrm{J} / \psi \mathrm{K}_{\mathrm{S}}\right)}{A\left(\mathrm{~B}^{0} \rightarrow \mathrm{J} / \psi \mathrm{K}_{\mathrm{S}}\right)}=-\frac{\left(V_{\mathrm{cb}}^{*} V_{\mathrm{cs}} V_{\mathrm{us}}^{*} V_{\mathrm{ud}}\right)^{2}}{\left|V_{\mathrm{cb}}^{*} V_{\mathrm{cs}} V_{\mathrm{us}}^{*} V_{\mathrm{ud}}\right|^{2}} .
$$

Using the formulae developed in the previous section, the time dependent rates for the initial $\mathrm{B}^{0}$ decaying into $\mathrm{J} / \psi \mathrm{K}_{\mathrm{S}}, R_{\mathrm{J} / \psi \mathrm{K}_{\mathrm{S}}}(t)$, and that for $\overline{\mathrm{B}}^{0}$ decaying into $\mathrm{J} / \psi \mathrm{K}_{\mathrm{S}}$, $\bar{R}_{\mathrm{J} / \psi \mathrm{K}_{\mathrm{S}}}(t)$ are given by

$$
\begin{aligned}
& R_{\mathrm{J} / \psi \mathrm{K}_{\mathrm{S}}}(t) \propto e^{-\hat{\Gamma} t}\left(1+\Im L_{\mathrm{J} / \psi \mathrm{K}_{\mathrm{S}}} \sin \Delta m t\right) \\
& \bar{R}_{\mathrm{J} / \psi \mathrm{K}_{\mathrm{S}}}(t) \propto e^{-\hat{\Gamma} t}\left(1-\Im L_{\mathrm{J} / \psi \mathrm{K}_{\mathrm{S}}} \sin \Delta m t\right)
\end{aligned}
$$

which allow to extract

$$
\Im L_{\mathrm{J} / \psi \mathrm{K}_{\mathrm{S}}}=\Im\left(\zeta \times \frac{A\left(\overline{\mathrm{B}}^{0} \rightarrow \mathrm{J} / \psi \mathrm{K}_{\mathrm{S}}\right)}{A\left(\mathrm{~B}^{0} \rightarrow \mathrm{J} / \psi \mathrm{K}_{\mathrm{S}}\right)}\right)=-\Im\left[\frac{\left(V_{\mathrm{td}}^{*} V_{\mathrm{tb}} V_{\mathrm{cb}}^{*} V_{\mathrm{cs}} V_{\mathrm{us}}^{*} V_{\mathrm{ud}}\right)^{2}}{\left|V_{\mathrm{td}}^{*} V_{\mathrm{tb}} V_{\mathrm{cb}}^{*} V_{\mathrm{cs}} V_{\mathrm{us}}^{*} V_{\mathrm{ud}}\right|^{2}}\right]
$$

With the Wolfenstein parameterization, it follows that

$$
\Im L_{\mathrm{J} / \psi \mathrm{K}_{\mathrm{S}}}=-\sin 2 \phi_{1}
$$

The same argument holds for the $\mathrm{B}_{\mathrm{s}} \rightarrow \mathrm{J} / \psi \phi$ decays and from the time dependent decay rates

$$
\begin{aligned}
R_{\mathrm{J} / \psi \phi}(t) & \propto e^{-\hat{\Gamma} t}\left(\cosh \frac{\Delta \Gamma}{2} t+2 \Re L_{\mathrm{J} / \psi \phi} \sinh \frac{\Delta \Gamma}{2} t+\Im L_{\mathrm{J} / \psi \phi} \sin \Delta m t\right) \\
\bar{R}_{\mathrm{J} / \psi \phi}(t) & \propto e^{-\hat{\Gamma} t}\left(\cosh \frac{\Delta \Gamma}{2} t+2 \Re L_{\mathrm{J} / \psi \phi} \sinh \frac{\Delta \Gamma}{2} t-\Im, L_{\mathrm{J} / \psi \phi} \sin \Delta m t\right)
\end{aligned}
$$

one can extract

$$
\Im L_{\mathrm{J} / \psi \phi}=\Im\left[\zeta \times \frac{A\left(\overline{\mathrm{B}}_{\mathrm{s}}^{0} \rightarrow \mathrm{J} / \psi \phi\right)}{A\left(\mathrm{~B}_{\mathrm{s}}^{0} \rightarrow \mathrm{J} / \psi \phi\right)}\right]=-\sin 2 \delta \phi_{3}
$$

Note that we assumed in the calculation above that $C P(\mathrm{~J} / \psi \phi)=+1$, i.e. the $\mathrm{J} / \psi \phi$ state is in the lowest orbital angular momentum state of $l=0$. If there exists 

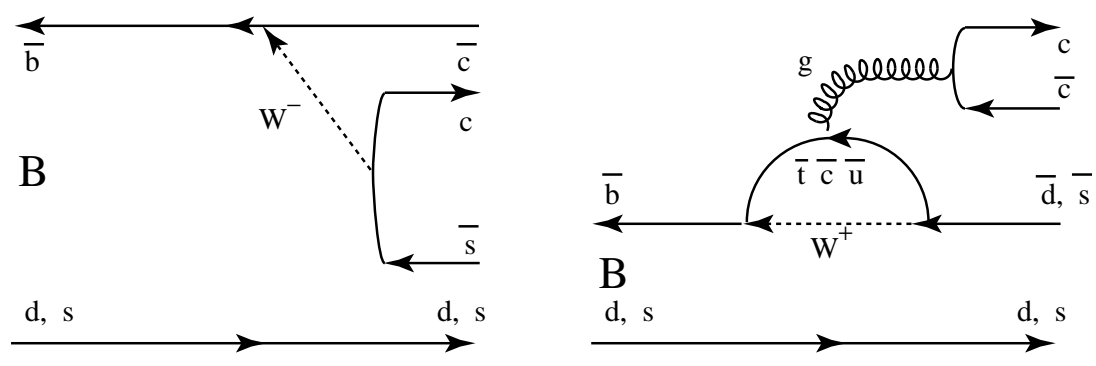

Figure 8: Tree and penguin diagrams contributing to the $\mathrm{B}^{0} \rightarrow \mathrm{J} / \psi \mathrm{K}_{\mathrm{S}}$ and $\mathrm{B}_{\mathrm{s}}^{0} \rightarrow$ $\mathrm{J} / \psi \phi$ decays.

the $l=1$ state with $C P(\mathrm{~J} / \psi \phi)=-1$, the measured $\Im L_{\mathrm{J} / \psi \phi}$ will be diluted and the fraction of the $C P=-1$ state must be experimentally measured. If there is the same amount of $C P=+1$ state and $C P=-1$ state, $\Im L_{\mathrm{J} / \psi \phi}$ will vanish.

An even cleaner decay channel is $\mathrm{B}^{0} \rightarrow \mathrm{D}^{* \mp} \pi^{ \pm}$. There is only one tree diagram, $\overline{\mathrm{b}} \rightarrow \overline{\mathrm{c}}+\mathrm{W}^{+}$followed by $\mathrm{W}^{+} \rightarrow \mathrm{u}+\overline{\mathrm{d}}$, which contributes to the $\mathrm{B}^{0} \rightarrow \mathrm{D}^{*-} \pi^{+}$decays. The same final state can be produced from the $\overline{\mathrm{B}}^{0}$ decays with another tree diagram, $\mathrm{b} \rightarrow \mathrm{u}+\mathrm{W}^{-}$followed by $\mathrm{W}^{-} \rightarrow \overline{\mathrm{c}}+\mathrm{d}$ (Figure $\mathrm{g}$ ). Therefore, the time dependent rate for the initial $\mathrm{B}^{0}$ decaying into $\mathrm{D}^{*-} \pi^{+}$is given by

$$
R_{\mathrm{D}^{*-}}(t) \propto e^{-\hat{\Gamma} t}\left[1+\frac{\left(1-\left|L_{\mathrm{D}^{*-} \pi^{+}}\right|^{2}\right)}{\left(1+\left|L_{\mathrm{D}^{*-} \pi^{+}}\right|^{2}\right)} \cos \Delta m t+\frac{2 \Im L_{\mathrm{D}^{*-} \pi^{+}}}{\left(1+\left|L_{\mathrm{D}^{*-} \pi^{+}}\right|^{2}\right)} \sin \Delta m t\right]
$$

where

$$
L_{\mathrm{D}^{*-} \pi^{+}}=\zeta \times \frac{A\left(\overline{\mathrm{B}}^{0} \rightarrow \mathrm{D}^{*-} \pi^{+}\right)}{A\left(\mathrm{~B}^{0} \rightarrow \mathrm{D}^{*-} \pi^{+}\right)}
$$

The weak phase of $A\left(\overline{\mathrm{B}}^{0} \rightarrow \mathrm{D}^{*-} \pi^{+}\right)$is given by $V_{\mathrm{ub}} V_{\mathrm{cd}}^{*}$ and that of $A\left(\mathrm{~B}^{0} \rightarrow \mathrm{D}^{*-} \pi^{+}\right)$ by $V_{\mathrm{cb}}^{*} V_{\mathrm{ud}}$. The phase of $L_{\mathrm{D}^{*-} \pi^{+}}$is then derived to be

$$
\begin{aligned}
\arg L_{\mathrm{D}^{*-} \pi^{+}} & =\arg V_{\mathrm{ub}}-\arg M_{12}+\varphi_{\mathrm{S}} \\
& =-\phi_{3}+2 \phi_{1}+\varphi_{\mathrm{S}}
\end{aligned}
$$

where $\varphi_{\mathrm{S}}$ is a possible strong phase difference between the $\mathrm{b} \rightarrow \mathrm{u}+\mathrm{W}^{-}$and $\overline{\mathrm{b}} \rightarrow$ $\overline{\mathrm{c}}+\mathrm{W}^{+}$tree diagrams.

CP conjugated decay amplitudes of $A\left(\overline{\mathrm{B}}^{0} \rightarrow \mathrm{D}^{*-} \pi^{+}\right)$and $A\left(\mathrm{~B}^{0} \rightarrow \mathrm{D}^{*-} \pi^{+}\right)$, i.e. $A\left(\mathrm{~B}^{0} \rightarrow \mathrm{D}^{*+} \pi^{-}\right)$and $A\left(\overline{\mathrm{B}}^{0} \rightarrow \mathrm{D}^{*+} \pi^{-}\right)$respectively, are obtained by taking the complex conjugate of the weak amplitudes while the strong phase remains unchanged. Thus for $\mathrm{D}^{*+} \pi^{-}$we obtain

$$
R_{\mathrm{D}^{*+}}(t) \propto e^{-\hat{\Gamma} t}\left[1-\frac{\left(1-\left|L_{\mathrm{D}^{*-} \pi^{+}}^{\mathrm{CP}}\right|^{2}\right)}{\left(1+\left|L_{\mathrm{D}^{*-} \pi^{+}}^{\mathrm{CP}}\right|^{2}\right)} \cos \Delta m t-\frac{2 \Im L_{\mathrm{D}^{*-\pi} \pi^{+}}^{\mathrm{CP}}}{\left(1+\left|L_{\mathrm{D}^{*-} \pi^{+}}^{\mathrm{CP}}\right|^{2}\right)} \sin \Delta m t\right]
$$



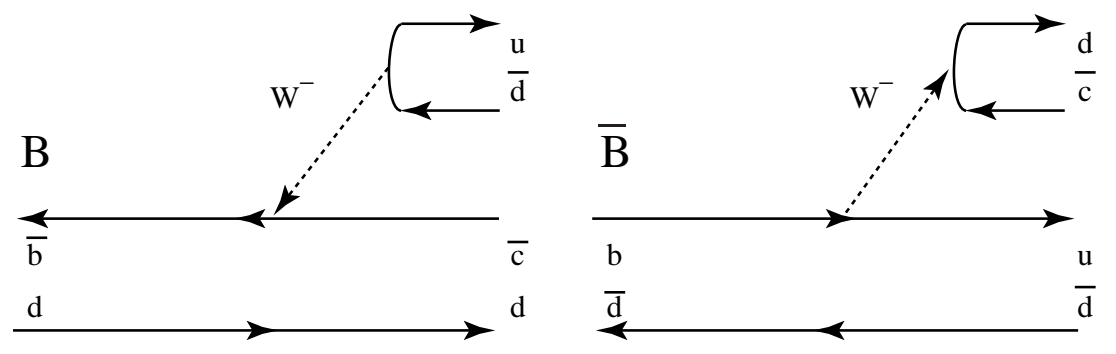

Figure 9: Tree diagrams contributing for the $\mathrm{B}^{0} \rightarrow \mathrm{D}^{*-} \pi^{+}$and $\overline{\mathrm{B}}^{0} \rightarrow \mathrm{D}^{*-} \pi^{+}$decays.

where

$$
L_{\mathrm{D}^{*-} \pi^{+}}^{\mathrm{CP}}=\frac{1}{\zeta} \times \frac{A\left(\mathrm{~B}^{0} \rightarrow \mathrm{D}^{*+} \pi^{-}\right)}{A\left(\overline{\mathrm{B}}^{0} \rightarrow \mathrm{D}^{*+} \pi^{-}\right)}
$$

and the phase of $L_{\mathrm{D}^{*-} \pi^{+}}^{\mathrm{CP}}$ is given by

$$
\begin{aligned}
\arg L_{D^{*-} \pi^{+}}^{\mathrm{CP}} & =-\arg V_{\mathrm{ub}}+\arg M_{12}+\varphi_{\mathrm{S}} \\
& =\phi_{3}-2 \phi_{1}+\varphi_{\mathrm{S}}
\end{aligned}
$$

From the two time dependent decay rates, we can extract $\phi_{3}-2 \phi_{1}$.

Note that

$$
\left|L_{\mathrm{D}^{*-} \pi^{+}}\right|=\left|L_{\mathrm{D}^{*-} \pi^{+}}^{\mathrm{CP}}\right| \approx\left|\frac{V_{\mathrm{ub}} V_{\mathrm{cd}}^{*}}{V_{\mathrm{cb}}^{*} V_{\mathrm{ud}}}\right|=\lambda^{2} \sqrt{\rho^{2} \eta^{2}}<<1
$$

i.e. the effect we have to measure is small.

The $\mathrm{CP}$ conjugated time dependent decay rate distributions are given by

$$
\bar{R}_{\mathrm{D}^{*+}}(t) \propto e^{-\hat{\Gamma} t}\left[1+\frac{\left(1-\left|L_{\mathrm{D}^{*-} \pi^{+}}^{\mathrm{CP}}\right|^{2}\right)}{\left(1+\left|L_{\mathrm{D}^{*-} \pi^{+}}^{\mathrm{CP}}\right|^{2}\right)} \cos \Delta m t+\frac{2 \Im L_{\mathrm{D}^{*-} \pi^{+}}^{\mathrm{CP}}}{\left(1+\left|L_{\mathrm{D}^{*-} \pi^{+}}^{\mathrm{CP}}\right|^{2}\right)} \sin \Delta m t\right]
$$

and

$$
\bar{R}_{\mathrm{D}^{*-}}(t) \propto e^{-\hat{\Gamma} t}\left[1-\frac{\left(1-\left|L_{\mathrm{D}^{*-} \pi^{+}}\right|^{2}\right)}{\left(1+\left|L_{\mathrm{D}^{*-} \pi^{+}}\right|^{2}\right)} \cos \Delta m t-\frac{2 \Im L_{\mathrm{D}^{*-} \pi^{+}}}{\left(1+\left|L_{\mathrm{D}^{*-} \pi^{+}}\right|^{2}\right)} \sin \Delta m t\right]
$$

which can be used to obtain the same information.

A similar method can be used for the $\mathrm{B}_{\mathrm{s}}^{0} \rightarrow \mathrm{D}_{\mathrm{s}}^{\mp} \mathrm{K}^{ \pm}$decays to extract $\phi_{3}-2 \delta \phi_{3}$. The effect is larger since

$$
\left|L_{\mathrm{D}_{\mathrm{s}}^{-} \mathrm{K}^{+}}\right| \approx\left|\frac{V_{\mathrm{ub}} V_{\mathrm{cs}}^{*}}{V_{\mathrm{cb}}^{*} V_{\mathrm{us}}}\right|=\sqrt{\rho^{2}+\eta^{2}}=O(1) .
$$




\subsubsection{CP Violation: Not So Clean Case}

The penguin contribution to the $\mathrm{B}_{\mathrm{d}} \rightarrow \pi^{+} \pi^{-}$decay was originally thought to be small and the decay would be dominated by the $\mathrm{b} \rightarrow \mathrm{u}+\mathrm{W}$ tree diagram. However, the discovery of $B\left(\mathrm{~B}_{\mathrm{d}} \rightarrow \mathrm{K}^{ \pm} \pi^{\mp}\right)>B\left(\mathrm{~B}_{\mathrm{d}} \rightarrow \pi^{+} \pi^{-}\right)$indicates that the contribution of the penguin diagrams to the $\mathrm{B}_{\mathrm{d}} \rightarrow \pi^{+} \pi^{-}$amplitude should be $\sim 20 \%$ or more.

Due to the penguin contribution, the phase of the $\mathrm{B}^{0} \rightarrow \pi^{+} \pi^{-}$decay amplitude deviates from that of $V_{\mathrm{ub}}^{*}$. Furthermore, CP violation in the decay amplitude could be present. Evaluation of those effects involves calculating contributions from different diagrams accurately. Strong interactions may play an important role as well. Therefore, this decay mode may not be ideal to make precise determinations of $\rho$ and $\eta$ from $\mathrm{CP}$ violation.

\subsection{Case with New Physics}

Decay processes where only the tree diagrams contribute should be unaffected by the presence of physics beyond the Standard Model. Therefore, $\left|V_{\mathrm{cb}}\right|$ and $\left|V_{\mathrm{ub}}\right|$ obtained from the semileptonic decays of $\mathrm{B}$ mesons would not be affected by the new physics and $A$ and $\rho^{2}+\eta^{2}$ can be obtained even if physics beyond the Standard Model is present.

New physics could generate $\mathrm{B}^{0}-\overline{\mathrm{B}}^{0}$ and $\mathrm{B}_{\mathrm{s}}^{0}-\overline{\mathrm{B}}_{\mathrm{s}}^{0}$ oscillations by new particles generating new box diagrams. They could also generate a tree level flavour changing neutral current contributing to the oscillation. Since these contributions are through "virtual" states, they contribute to $M_{12}$ with little effect on $\Gamma_{12}$, i.e.

$$
M_{12}=M_{12}^{\mathrm{SM}}+M_{12}^{\mathrm{NP}}, \Gamma_{12}=\Gamma_{12}^{\mathrm{SM}}
$$

where $M_{12}^{\mathrm{SM}}$ and $\Gamma_{12}^{\mathrm{SM}}$ are due to the Standard Model and $M_{12}^{\mathrm{NP}}$ is the contribution from the new physics. The measured $\Delta m$ is given by $2\left|M_{12}\right|$ and can no longer used to extract $\left|V_{\mathrm{td}}\right|^{2}$ due to $M_{12}^{\mathrm{NP}}$.

Since

$$
\left|\frac{\Gamma_{12}}{M_{12}}\right|=\frac{2\left|\Gamma_{12}^{\mathrm{SM}}\right|}{\Delta m}
$$

remains small, CP violation in the oscillation remains small as seen from equation 31 . Therefore,

$$
\zeta=e^{-i \varphi_{M}}
$$

is still valid. However, note that

$$
\varphi_{M} \equiv \arg M_{12} \neq \arg M_{12}^{\mathrm{SM}}
$$


Decay amplitudes from the penguin diagrams can be affected by physics beyond the Standard Model since new particles can contribute virtually in the loop. Therefore, the modes such as $\mathrm{B}_{\mathrm{d}}$ decaying into $\pi^{+} \pi^{-}, \mathrm{K}^{ \pm} \pi^{\mp}$ may have some contribution from the new physics.

Since the decays $\mathrm{B}_{\mathrm{d}} \rightarrow \mathrm{J} / \psi \mathrm{K}_{\mathrm{S}}$ and $\mathrm{B}_{\mathrm{s}} \rightarrow \mathrm{J} / \psi \phi$ are tree dominated, they are little affected by new physics. Therefore we have

$$
\frac{A\left(\overline{\mathrm{B}}^{0} \rightarrow \mathrm{J} / \psi \mathrm{K}_{\mathrm{S}}\right)}{A\left(\mathrm{~B}^{0} \rightarrow \mathrm{J} / \psi \mathrm{K}_{\mathrm{S}}\right)}=-\frac{A\left(\mathrm{~B}_{\mathrm{s}}^{0} \rightarrow \mathrm{J} / \psi \phi\right)}{A\left(\overline{\mathrm{B}}_{\mathrm{s}}^{0} \rightarrow \mathrm{J} / \psi \phi\right)}=-1
$$

with the phase convention due to the Wolfenstein parameterization and

$$
L_{\mathrm{J} / \psi \mathrm{K}_{\mathrm{S}}, \mathrm{J} / \psi \phi}=\mp e^{-i \varphi_{M}}:- \text { for } \mathrm{B}_{\mathrm{d}} \rightarrow \mathrm{J} / \psi \mathrm{K}_{\mathrm{S}} \text { and }+ \text { for } \mathrm{B}_{\mathrm{s}} \rightarrow \mathrm{J} / \psi \phi
$$

and studies of the time dependent decay rates give $\arg M_{12}$.

The $\mathrm{B}_{\mathrm{d}} \rightarrow \mathrm{D}^{*} \pi$ and $\mathrm{B}_{\mathrm{s}} \rightarrow \mathrm{D}_{\mathrm{s}} \mathrm{K}$ decays are generated by only the tree diagrams and not affected by new physics. Therefore we have

$$
\arg L_{\mathrm{D}^{*-} \pi^{+}}=-\phi_{3}-\arg M_{12}+\varphi_{\mathrm{S}}
$$

and

$$
\arg L_{\mathrm{D}^{*+} \pi^{-}}=\phi_{3}+\arg M_{12}+\varphi_{\mathrm{S}}
$$

and studies of the time dependent decay rates provide $\arg M_{12}+\phi_{3}$. Similarly studies can be done for $\mathrm{B}_{\mathrm{s}} \rightarrow \mathrm{D}_{\mathrm{s}} \mathrm{K}$.

By combining the measurements of $\mathrm{B}_{\mathrm{d}} \rightarrow \mathrm{J} / \psi \mathrm{K}_{\mathrm{S}}$ and $\rightarrow \mathrm{D}^{*} \pi$ or $\mathrm{B}_{\mathrm{s}} \rightarrow \mathrm{J} / \psi \phi$ and $\rightarrow \mathrm{D}_{\mathrm{s}} \mathrm{K}$, the angle $\phi_{3}$ can be determined even with presence of physics beyond the Standard Model. By comparing the result from $\mathrm{B}_{\mathrm{d}}$ and that from $\mathrm{B}_{\mathrm{s}}$, consistency of the method can be tested. Since the phase of $V_{\mathrm{ub}}$ is given by $\phi_{3}$ and its modulus is measured from the semileptonic decay, $\rho$ and $\eta$ can be extracted. Once $\lambda, A, \rho$ and $\eta$ are known, $M_{12}^{\mathrm{SM}}$ can be calculated and from the measured $\Delta m$ and $\arg M_{12}$, the new physics contribution $M_{12}^{\mathrm{NP}}$ is obtained. This can be used to identify the nature of the new physics contributing to the oscillation.

\subsection{Experimental Prospects}

A possible experimental programme for the study of $\mathrm{CP}$ violation in the $\mathrm{B}$ meson system and search for physics beyond the Standard Model can be summarised in the following steps:

1. Determination of $\left|V_{\mathrm{cb}}\right|$ and $\left|V_{\mathrm{ub}}\right|$ from semileptonic (and some hadronic) decays.

2. Measurement of $\Delta m$ for $\mathrm{B}_{\mathrm{d}}$ and $\mathrm{B}_{\mathrm{s}}$ 
3. Measurement of $\Im L_{\mathrm{J} / \psi \mathrm{K}_{\mathrm{S}}}$

4. Measurement of $L_{\mathrm{J} / \psi \phi}, L_{\mathrm{D}^{*} \mp \pi^{ \pm}}$and $L_{\mathrm{D}_{\mathrm{s}}^{\mp} \mathrm{K}^{ \pm}}$

The first step has been made by ARGUS and CLEO at $\Upsilon(4 S)$ machines and the four LEP experiments. BABAR and BELLE at the new asymmetric $\Upsilon(4 S)$ machines and CLEO will improve the precisions on those determinations. Future improvement of theory is also an important factor. Half of the second step, $\Delta m\left(\mathrm{~B}_{\mathrm{d}}\right)$ was done by ARGUS, CLEO, UA1 at the SPS Collider, the four LEP experiments, SLD at $\mathrm{SLC}$ and $\mathrm{CDF}$ at the Tevatron. For $\Delta m\left(\mathrm{~B}_{\mathrm{s}}\right)$, we may have to wait for the next data taking by CDF, D0 and HERA-B. The third step will be made by BABAR, BELLE, CDF, D0 and possibly HERA-B by the year 2005 .

After the second step, four parameters of the CKM matrix are all defined within the framework of the Standard Model, e.g. $A, \lambda, \rho$ and $\eta$. The third step provides an additional information $\tan ^{-1} \eta /(1-\rho)$ within the framework of the Standard Model and consistency of the CKM picture can now be tested.

As demonstrated in the previous chapter, if physics beyond the Standard Model exists, the fourth step is needed to clearly establish the evidence of new physics and separate the effect due to the Standard Model and that from new physics. After the third step, only $\rho^{2}+\eta^{2}$ will be known from $\left|V_{\mathrm{ub}}\right|$ and the information on $\tan ^{-1} \eta /(1-\rho)$ is spoiled by new physics. Only after the fourth step, $\rho$ and $\eta$ can be determined, together with isolating the new physics contribution.

For the last step, new generation of experiments with statistics much higher than $10^{10} \mathrm{~B}$ mesons are needed. The $\mathrm{B}_{\mathrm{s}}$ meson is an essential ingredient. After 2005, LHC will be the most powerful source of B mesons. Experiments must be equipped with a trigger efficient for hadronic decay modes to gain high statistics for the necessary final states. Particle identification is also crucial in order to reduce background. $\mathrm{LHCb}$ is a detector at the $\mathrm{LHC}$ optimised for $\mathrm{CP}$ violation studies with $\mathrm{B}$ mesons. The two general purpose LHC detectors, ATLAS and CMS can contribute only to a limited aspect of the fourth step. A proposed experiment at Tevatron, BTeV, can also make the last two steps.

Clearly CP violation is expected in many other decay channels. For many of them, there are some theoretical problems for making accurate predictions. However, they can be used to make a systematic study which will provide a global picture whether $\mathrm{CP}$ violation can fit into the CKM picture. With all those experiments, we continue to improve our understanding of $\mathrm{CP}$ violation and hope to discover physics beyond the Standard Model. 


\section{Acknowledgement}

The author is very grateful to the organizers of this school for their extended hospitality and efforts to prepare such a stimulating environment. R. Forty is acknowledged for reading this manuscript and giving many useful comments. The author appreciates many useful comments by O. Schneider.

\section{References}

[1] C. S. Wu et al., Phys. Rev. 105 (1957) 1413.

[2] R.L Garwin et al., Phys. Rev. 105 (1957) 1415, J. I. Friedman and V. L. Telegdi, Phys. Rev. 105 (1957) 1681.

[3] M. Gell-Mann and R. P. Feynmann, Phys. Rev. 109 (1958) 193, E. C. G. Sudarshan and R. E. Marshak, Phys. Rev. 109 (1958) 1860, J. Sakurai, Nuovo Cim. 7 (1958) 649.

[4] J. H. Christenson et al., Phys Rev. Lett. 13 (1964) 138.

[5] N. Cabibbo, Phys. Rev. Lett. 10 (1963) 531, M. Kobayashi and K. Maskawa, Prog. Theor. Phys. 49 (1972) 282.

[6] A.D. Sakharov, JETP Lett. 6 (1967) 21.

[7] See for example M.B. Gavela et al., Modern Phys. Lett. 9A (1994) 795.

[8] V. Weisskopf and E. Wigner, Z. fúr Physik, 63 (1930) 54.

[9] For pioneering works see

A. Pais and S. B. Treiman, Phys. Rev. D12 (1975) 2744,

L. B. Okun et al, Lett. Nuovo Cimento 13 (1975) 218, M. Bander et al, Phys. Rev. Lett. 43 (1979) 242,

A. B. Carter and A. I. Sanda, Phys. Rev. D23 (1981) 1567, I. I. Bigi and A. I. Sanda, Nucl. Phys. B193 (1981) 85.

[10] More details can be found in

T. Nakada, CP Violation in K- and B-Meson Decays, PSI-PR-91-02, 1991.

[11] G. Lüders, Dan. Mat. Fys. Medd. 28, No5 (1954),

W. Pauli, In Niels Bohr and the development of physics, ed. W. Pauli, pp. 30, New York, Pergamon Press, 1995,

R. Jost, Helv. Phys. Acta, 30 (1975) 409,

G. Lüder, Annals of Physics 2 (1957) 1. 
[12] C. Caso et al, The European Physical Journal C3 (1998) 1 and 1999 off-year partial update for the 2000 edition available on the PDG WWW pages (URL: http://pdg.lbl.gov/).

[13] A. Angelopoulos et al. [CPLEAR Collaboration], Phys. Lett. B444 (1998) 43.

[14] A. Apostolakis et al. [CPLEAR Collaboration], Phys. Lett. B458 (1999) 545.

[15] W. Ochs, MPI-Ph/Ph 91-35, (1991).

[16] G. D. Barr et al. [NA31 Collaboration], system," Phys. Lett. B317 (1993) 233.

[17] L. K. Gibbons et al., epsilon)," Phys. Rev. Lett. 70 (1993) 1203.

[18] A. Alavi-Harati et al. [KTeV Collaboration], Phys. Rev. Lett. 83 (1999) 22.

[19] V. Fanti et al. [NA48 Collaboration], neutral kaon," Phys. Lett. B465 (1999) 335.

[20] For details of the Standard Model description of the K and B system and further references, see the following articles:

A. J. Buras, Weak Hamiltonian, CP violation and rare decays, hep-ph/9806471, A. J. Buras, CP violation and rare decays of $K$ and $B$ mesons, hep-ph/9905437.

[21] L. Wolfenstein, Phys. Rev. Lett. 51 (1983) 1945.

[22] A. Alavi-Harati et al. [The E799-II/KTeV Collaboration], gamma," hepex/9907014. 